Research paper

\title{
Stochastic modelling of slow-progressing tumors: Analysis and applications to the cell interplay and control of low grade gliomas
}

\author{
Clara Rojas Rodríguez ${ }^{\mathrm{a}}$, Gabriel Fernández Calvo ${ }^{\mathrm{a}}$, Ignacio Ramis-Conde ${ }^{\mathrm{b}}$, \\ Juan Belmonte-Beitia ${ }^{\mathrm{a}, *}$ \\ a Department of Mathematics and Mathematical Oncology Laboratory, University of Castilla-La Mancha, 13071 Ciudad Real, Spain \\ ${ }^{\mathrm{b}}$ Department of Mathematics and Mathematical Oncology Laboratory, Faculty of Education, University of Castilla-La Mancha, Cuenca, \\ Spain
}

\section{A R T I C L E I N F O}

\section{Article history:}

Received 25 September 2016

Revised 9 January 2017

Accepted 7 February 2017

Available online 8 February 2017

\section{Keywords:}

Tumor growth

Optimal control

Stochastic master equations

Stability analysis

Gliomas

Chemotherapy

\begin{abstract}
A B S T R A C T
Tumor-normal cell interplay defines the course of a neoplastic malignancy. The outcome of this dual relation is the ultimate prevailing of one of the cells and the death or retreat of the other. In this paper we study the mathematical principles that underlay one important scenario: that of slow-progressing cancers. For this, we develop, within a stochastic framework, a mathematical model to account for tumor-normal cell interaction in such a clinically relevant situation and derive a number of deterministic approximations from the stochastic model. We consider in detail the existence and uniqueness of the solutions of the deterministic model and study the stability analysis. We then focus our model to the specific case of low grade gliomas, where we introduce an optimal control problem for different objective functionals under the administration of chemotherapy. We derive the conditions for which singular and bang-bang control exist and calculate the optimal control and states.
\end{abstract}

(c) 2017 Elsevier B.V. All rights reserved.

\section{Introduction}

Stochastic processes are ubiquitous in biological systems [2,10,16]. Important examples range from gene expression [48], cellular differentiation [41], to animal migration and infectious disease risk [3]. The interface of malignant cancer cells with the surrounding healthy tissue provides another relevant scenario for stochastic processes [4,15,19,23,24,30,31,47,63]. Disruption of the balance between early formed neoplastic cells and the normal cells results in the invasion and dissemination of the malignant cells and eventually a transition from a benign to a malignant tumor. This cell to cell interaction is crucial as it defines the basis of later advanced cancer stages: different pathways at the initial phases of growth will produce dramatically different natural histories of the tumor, which will be reflected not only in the distinct morphologies displayed $[6,22,56]$ but also in the diversity of responses to therapies [35].

Another layer of complexity is the relative speed at which tumors advance. Tumors that exhibit a fast progression are characterized by a strong competition for resources; this includes access to space, oxygen and nutrients. Thus, a considerable

\footnotetext{
* Corresponding author.

E-mail addresses: Juan.Belmonte@uclm.es, juan.belmonte@uclm.es (J. Belmonte-Beitia).
} 
restructuration of the pre-existing healthy tissue occurs, typically resulting in the growth of a vascular network, which is often aberrant and dysfunctional, and in the development of necrotic regions [1]. The modification of the spatial environment re-defines the malignant-normal cell interaction that initially governed the system [18,56]. As a consequence, the conditions that were present during tumorigenesis change dramatically and may become masked by the complexity of a fast-evolving and multi-variable system. From a therapeutical point of view, modelling this situation poses enormous challenges.

In contrast, focusing the attention on slow-progressing tumors, a somewhat more localized interplay between cells prevails for relatively longer times, so that the marked spatio-temporal complexity encountered in fast-growing tumors is less pronounced. Of course, in the long term, if the tumor becomes sufficiently large, then such a localized interaction between (malignant and normal) cells cannot be maintained.

Modelling slow-progressing tumors, although perhaps less demanding from a mathematical and computational point of view, is nonetheless relevant from a clinical point of view. It should be stressed that slow progression does not necessarily imply less malignancy. There are a number of cancer types that can grow slowly for a significant fraction of their natural history and yet have a dismal prognosis. For instance, pancreatic cancers may require up to 29 months to achieve a size of $1 \mathrm{~cm}$ [49], very rarely demonstrating any specific symptoms (thus making them quite difficult to detect), and still remain lethal if treatment is delayed. Another important example are low grade gliomas.

The World Health Organization (WHO) classifies the tumors of the central nervous system by their cell of origin, histopathological characteristics and molecular signatures, which are predictive of their progression [42,43]. Gliomas are neuroepithelial tumors originating from the supporting glial cells of the central nervous system. The most frequent gliomas comprise diffuse astrocytic and oligodendroglial tumors. Low grade gliomas (LLG) encompass a heterogeneous group of primary brain tumors (WHO grade I and II) that are typically well-differentiated, do not exhibit prominent histologic features [27,61], and account for approximately 15\% of all gliomas [36]. These tumors often arise in young, but otherwise healthy patients, and generally present an asymptomatic course with a lower incidence and a longer-term survival when compared with high-grade gliomas (median overall survival drops from about 7 years for grade II to 15 months for grade IV). Most of these tumors very rarely, if ever, metastasize outside the brain. Treatment options include observation, surgery, radiation, chemotherapy, and combined modalities, which are based on tumor location, histology, molecular profile and patient characteristics $[27,61]$.

The timing of treatment in LGG remains a controversial issue. A number of works support early intervention, including surgical resection, radiotherapy and chemotherapy [61]. Chemotherapy has been traditionally utilized as a last-resort alternative following surgery and radiotherapy, once tumor recurrence is observed. The most studied chemotherapies are PVC (combination of procarbazine, lomustine and vincristine) and temozolomide. PVC is usually administered in cycles of approximately 8 weeks [27,61]. Since LGG are inevitably fatal in the long term; in most cases the tumor progresses towards a higher grade glioma (WHO grade III and IV), it is an open question how to "best" administer chemotherapy to maintain a prolonged control of the tumor (maximize the free progression survival) and reduce as much as possible neurocognitive toxicities in the patient. From a mathematical point of view this problem motivates to carefully define both the key ingredients governing the (necessarily) stochastic interaction between tumor and normal cells and the introduction of a suitable optimization control problem to model the treatment response.

Therefore, the main purpose of this paper is dual: firstly, building from a continuum stochastic non-spatial model, we derive a minimal deterministic model that accounts for the tumor-normal cell interplay under slow progression, which is later focused on low-grade gliomas. Secondly, we consider the response of our tumor model to the administration of chemotherapy. To this end, we apply optimal control theory to find optimal therapy strategies within our model, calculating bang-bang and singular optimal controls.

Thus, the paper is organized as follows. In Section 2 we introduce the mathematical model and discuss its biological meaning. Next, in Section 3 we carry out an expansion of the master equation to derive a system of deterministic equations. In Section 4 we study different mathematical properties of the continuous model and the stability of the corresponding stationary states. Moreover, we analyze the behaviour of the first and second moments of the fluctuations near the stationary state. Section 5 deals with the formulation of the optimal control problems using several objective functionals. After proving the existence of optimal controls, we discuss the necessary optimality conditions of Pontryagin's Maximum (Minimum) Principle [54]. Since the control variable appears linearly in the dynamic system and objectives, optimal controls are concatenations of bang-bang and singular arcs. We derive a formula for the singular control in terms of state and adjoint variables. A further necessary condition is the generalized Legendre-Clebsch condition [34,59] which we evaluate explicitly. The numerical simulations performed to solve the optimal control problems are based on dicretization techniques and nonlinear programming methods. Finally, in Section 6 we summarize our conclusions.

\section{The model}

We model the interaction between tumor and normal cells as a continuous-time bivariate Markov process [32]. Let $n_{i}$, with $i=1,2$, denote the number of tumor and normal cells, respectively. The initial number of tumor and normal cells will be represented by $\hat{n}_{i}\left(t_{0}\right) \neq 0$ and, without loss of generality, we take for the initial time $t_{0}=0$. Therefore, the continuoustime bivariate Markov process $\{X(t), Y(t): t \geq 0\}$ is defined on the space $S=\left\{\left(n_{1}, n_{2}\right): n_{1}, n_{2}=0,1,2 \ldots\right\}$. We assume that transitions can only occur between adjacent states. Therefore, since there are two random variables, $X(t)$ and $Y(t)$, we shall 
be concerned with the joint probability distribution at time $t$, given by

$$
P(X(t)=j, Y(t)=i)=p_{i j}(t) .
$$

Next, the conditional probability distribution of the relevant transitions in the interval $\Delta t$, from state $n=\left(n_{1}, n_{2}\right) \in S$ to state $m=\left(m_{1}, m_{2}\right) \in S$, reads as

$$
P\left(X(t+\Delta t)=m_{1}, Y(t+\Delta t)=m_{2} \mid X(t)=n_{1}, Y(t)=n_{2}\right)=p_{n m}(\Delta t) .
$$

In the limit $\Delta t \rightarrow 0^{+}$, the transition probabilities can be defined as

- $p_{n m}(\Delta t)=\alpha_{n_{1}, n_{2}}$, for $m=\left(n_{1}+1, n_{2}\right)$.

- $p_{n m}(\Delta t)=\bar{\alpha}_{n_{1}, n_{2}}$, for $m=\left(n_{1}, n_{2}+1\right)$.

- $p_{n m}(\Delta t)=\beta_{n_{1}, n_{2}}+\gamma_{n_{1}, n_{2}}+\mu_{n_{1}, n_{2}}^{(1)}$, for $m=\left(n_{1}-1, n_{2}\right)$.

- $p_{n m}(\Delta t)=\mu_{n_{1}, n_{2}}^{(2)}$, for $m=\left(n_{1}, n_{2}-1\right)$.

- $p_{n m}(\Delta t)=1-\left(\alpha_{n_{1}, n_{2}}+\bar{\alpha}_{n_{1}, n_{2}}+\beta_{n_{1}, n_{2}}+\gamma_{n_{1}, n_{2}}+\mu_{n_{1}, n_{2}}^{(1)}+\mu_{n_{1}, n_{2}}^{(2)}\right)$, for $m=\left(n_{1}, n_{2}\right)$.

- $p_{n m}(\Delta t)=o(\Delta t)$, otherwise, where $\lim _{\Delta t \rightarrow 0^{+}} \frac{f(t)}{\Delta t}=0$.

We define $\alpha_{n_{1}, n_{2}}$ and $\bar{\alpha}_{n_{1}, n_{2}}$ as the birth rates of tumor and healthy cells, respectively, and $\beta_{n_{1}, n_{2}}$ is the death rate of tumor cells. To account for resource (nutrients and oxygen) competition between tumor cells, we include the rate $\gamma_{n_{1}, n_{2}}$. Analogously, $\mu_{n_{1}, n_{2}}^{(1)}$ is the interaction rate between tumor and normal cells. Finally, the death rate for normal cells due to the presence of cancer cells is denoted by $\mu_{n_{1} n_{2}}^{(2)}$.

The first assumption of our model is that the healthy brain cells do not undergo mitosis, i.e., $\bar{\alpha}_{n_{1}, n_{2}}=0$, since the normal (adult) brain tissue (gray and white matter) does not exhibit a significant proliferation. Moreover, we set $\alpha_{0, j}=\beta_{0, j}=\gamma_{0, j}=$ $\mu_{0, j}^{(i)}=0$, with $i=1,2$, for $j \geq 0$.

Let $p_{n_{1}, n_{2}}$ denote the conditional probability that there are $n_{1}$ tumor cells and $n_{2}$ normal cells at time $t$, that is

$$
p_{n_{1}, n_{2}}=P\left(X(t)=n_{1}, Y(t)=n_{2} \mid X(0)=\hat{n}_{1}, Y(0)=\hat{n}_{2}\right),
$$

with $\sum_{n_{1}=0}^{\infty} \sum_{n_{2}=0}^{\infty} p_{n_{1}, n_{2}}(t)=1$. We resort to the theoretical framework of continuous-time Markov chains [2]. The forward Kolmogorov equation reads as

$$
\begin{aligned}
\frac{d p_{n_{1}, n_{2}}(t)}{d t}= & \alpha_{n_{1}-1, n_{2}} p_{n_{1}-1, n_{2}}(t)+\beta_{n_{1}+1, n_{2}} p_{n_{1}+1, n_{2}}(t)+\gamma_{n_{1}+1, n_{2}} p_{n_{1}+1, n_{2}}(t) \\
& +\mu_{n_{1}, n_{2}+1}^{(2)} p_{n_{1}, n_{2}+1}(t)+\mu_{n_{1}+1, n_{2}}^{(1)} p_{n_{1}+1, n_{2}}(t),
\end{aligned}
$$

together with

$$
\frac{d p_{0, n_{2}}}{d t}=\beta_{0, n_{2}} p_{1, n_{2}}
$$

Notice that the set $\left\{\left(n_{1}, n_{2}\right) \in S: n_{1}=0, n_{2} \neq 0\right\}$ is an absorbing set. Henceforth we will assume that the rates defined above satisfy the following conditions:

- The birth and death rates for the tumor cells can be written as $\alpha_{n_{1}, n_{2}}=\alpha n_{1}$ and $\beta_{n_{1}, n_{2}}=\beta n_{1}$, that is, independent on the number of normal cells. This assumption relies on the fact that the presence of the normal brain cells only affects the tumor cells via the competition for nutrients and oxygen, already taken into account.

- The death rate for the normal cells in the presence of the tumor cells is written in the form $\mu_{n_{1}, n_{2}}^{(2)}=\bar{\mu}^{(2)} g\left(n_{1}, n_{2}\right)$, where $g\left(n_{1}, n_{2}\right)$ is a positive homogeneous function of degree two. That is, $g\left(\eta n_{1}, \eta n_{2}\right)=\eta^{2} g\left(n_{1}, n_{2}\right)$, for any non-negative real $n_{1}, n_{2}$ and $\eta$. Therefore, we are assuming that the death of normal cells is mediated by a contact interaction term $g\left(n_{1}\right.$, $n_{2}$ ) between the tumor and the normal cells, with a death rate $\mu^{(2)}$. In general, the details of this interaction may be very complicated. In the case of LGG, from histopathology observations, the tumor (e.g., diffuse astrocytoma) cells consist of well differentiated fibrillary and gemistocytic neoplastic astrocytes on the background of a microcystic extracellular matrix [42]. Although these cells do not disrupt the vasculature, they vary considerably with respect to their size and functionality displaying an altered metabolism and signaling which affects the homeostasis of the normal brain cells.

- $\gamma_{n_{1}, n_{2}}=\bar{\gamma} n_{1} n_{1}$ and $\mu_{n_{1}, n_{2}}^{(1)}=\bar{\mu}^{(1)} n_{1} n_{2}$ are, respectively, the competition terms rate between tumor-tumor and tumornormal cells.

\section{Expansion of the master equation: derivation of the deterministic equations}

We start by noticing that Eq. (2) cannot be solved exactly. In fact, only in very few cases is the master equation amenable for explicit solutions. Therefore, we will resort to an expansion of the master Eq. (2) which will yield a macroscopic and deterministic equation capturing the average features of the stochastic model [32]. Firstly, let us introduce the following step operators, for any general function $f$ defined in $S$, which are given by:

$$
E_{n_{1}} f\left(n_{1}, n_{2}\right)=f\left(n_{1}+1, n_{2}\right) \text {, }
$$




$$
\begin{aligned}
E_{n_{2}} f\left(n_{1}, n_{2}\right) & =f\left(n_{1}, n_{2}+1\right), \\
E_{n_{1}}^{-1} f\left(n_{1}, n_{2}\right) & =f\left(n_{1}-1, n_{2}\right) .
\end{aligned}
$$

With the aid of these operators, the master Eq. (2) may be rewritten as

$$
\frac{d p_{n_{1}, n_{2}}(t)}{d t}=\left(E_{n_{1}}^{-1}-1\right) \alpha_{n_{1}, n_{2}} p_{n_{1}, n_{2}}+\left(E_{n_{1}}-1\right)\left[\beta_{n_{1}, n_{2}}+\gamma_{n_{1}, n_{2}}+\mu_{n_{1}, n_{2}}^{(1)}\right] p_{n_{1}, n_{2}}+\left(E_{n_{2}}-1\right) \mu_{n_{1}, n_{2}}^{(2)} p_{n_{1}, n_{2}} .
$$

Our next task is to introduce a suitable expansion parameter. Let $\Omega$ be this parameter, which measures the size (e.g., the volume) of the system. In the case of LGG it represents a sufficiently large radiologic volume that contains the tumor and the surrounding oedema, observable in T2-weighted or in Fluid-attenuated inversion-recovery (FLAIR) MRI [58]. We assume that if the total number of cells is large enough, then the fluctuations are relatively small and, therefore, the jumps are also small. Thus, the number of cells can be described as a sum of a deterministic density plus a small fluctuation, with the parameter $\Omega$ creating a distinction between both scales; macroscopic and fluctuation scales.

Let us denote by $x_{i}(t)$, with $i=1,2$, the deterministic densities of tumor and normal cells and by $\xi_{i}(t), i=1,2$ their fluctuations. We define the following relation:

$$
n_{i}=\Omega x_{i}+\Omega^{1 / 2} \xi_{i}=\Omega\left(x_{i}+\frac{1}{\Omega^{1 / 2}} \xi_{i}\right), \text { for } i=1,2,
$$

where the first term is macroscopic and the second term characterizes the fluctuations in the number of cells. We are assuming that the fluctuations are of the order $\Omega^{1 / 2}[32]$.

Eq. (6) is a time-dependent transformation from the variables $\left(n_{1}, n_{2}\right)$ to $\left(\xi_{1}, \xi_{2}\right)$ involving the yet undetermined functions $\left(x_{1}, x_{2}\right)$. With the above definition (6), the probability distribution $p_{n_{1}, n_{2}}$ transforms into a probability distribution $\Pi$ of $\xi_{1}$ and $\xi_{2}$,

$$
p_{n_{1}, n_{2}}(t)=p_{\Omega x_{1}+\Omega^{1 / 2} \xi_{1}, \Omega x_{2}+\Omega^{1 / 2} \xi_{2}}(t)=\Pi\left(\xi_{1}, \xi_{2}, t\right) .
$$

It then follows that

$$
\frac{\partial}{\partial \xi_{i}}=\Omega^{\frac{1}{2}} \frac{\partial}{\partial n_{i}} \text { and } \frac{\partial}{\partial \xi_{i}^{2}}=\Omega \frac{\partial}{\partial n_{i}^{2}} .
$$

Differentiating $n_{i}(t)$, for each $i=1,2$, we get

$$
\frac{d n_{i}}{d t}=\Omega \frac{d x_{i}}{d t}+\Omega^{\frac{1}{2}} \frac{d \xi_{i}}{d t}, \quad \text { for } i=1,2 .
$$

Therefore,

$$
\frac{d p}{d t}=\frac{\partial \Pi}{\partial t}-\Omega^{\frac{1}{2}} \frac{d x_{1}}{d t} \frac{\partial \Pi}{\partial \xi_{1}}-\Omega^{\frac{1}{2}} \frac{d x_{2}}{d t} \frac{\partial \Pi}{\partial \xi_{2}},
$$

where we have neglected terms of the order $O\left(\Omega^{-1 / 2}\right)$. Next, we Taylor expand the step operators (4)

$$
\begin{aligned}
& E_{n_{i}}=1+\Omega^{-1 / 2} \frac{\partial}{\partial \xi_{i}}+\frac{1}{2} \Omega^{-1} \frac{\partial^{2}}{\partial \xi_{i}^{2}}+O\left(\Omega^{-1}\right), \quad \text { for } i=1,2, \\
& E_{n_{1}}^{-1}=1-\Omega^{-1 / 2} \frac{\partial}{\partial \xi_{1}}+\frac{1}{2} \Omega^{-1} \frac{\partial^{2}}{\partial \xi_{1}^{2}}+O\left(\Omega^{-1}\right) .
\end{aligned}
$$

Consequently the forward Kolmogorov Eq. (2) (or master equation) in the new variables takes the form

$$
\begin{aligned}
\frac{\partial \Pi}{\partial t}-\Omega^{\frac{1}{2}}\left(\frac{d x_{1}}{d t} \frac{\partial \Pi}{\partial \xi_{1}}+\frac{d x_{2}}{d t} \frac{\partial \Pi}{\partial \xi_{2}}\right)= & \left(-\Omega^{-1 / 2} \frac{\partial}{\partial \xi_{1}}+\frac{1}{2} \Omega^{-1} \frac{\partial^{2}}{\partial \xi_{1}^{2}}\right) \alpha_{\Omega x_{1}+\Omega^{1 / 2} \xi_{1}, \Omega x_{2}+\Omega^{1 / 2} \xi_{2}} \Pi \\
& +\left(\Omega^{-1 / 2} \frac{\partial}{\partial \xi_{1}}+\frac{1}{2} \Omega^{-1} \frac{\partial^{2}}{\partial \xi_{1}^{2}}\right) \beta_{\Omega x_{1}+\Omega^{1 / 2} \xi_{1}, \Omega x_{2}+\Omega^{1 / 2} \xi_{2}} \Pi \\
& +\left(\Omega^{-1 / 2} \frac{\partial}{\partial \xi_{1}}+\frac{1}{2} \Omega^{-1} \frac{\partial^{2}}{\partial \xi_{1}^{2}}\right) \gamma_{\Omega x_{1}+\Omega^{1 / 2} \xi_{1}, \Omega x_{2}+\Omega^{1 / 2} \xi_{2}} \Pi \\
& +\left(\Omega^{-1 / 2} \frac{\partial}{\partial \xi_{1}}+\frac{1}{2} \Omega^{-1} \frac{\partial^{2}}{\partial \xi_{1}^{2}}\right) \mu_{\Omega x_{1}+\Omega^{1 / 2} \xi_{1}, \Omega x_{2}+\Omega^{1 / 2} \xi_{2}}^{(1)} \Pi \\
& +\left(\Omega^{-1 / 2} \frac{\partial}{\partial \xi_{2}}+\frac{1}{2} \Omega^{-1} \frac{\partial^{2}}{\partial \xi_{2}^{2}}\right) \mu_{\Omega x_{1}+\Omega^{1 / 2} \xi_{1}, \Omega x_{2}+\Omega^{1 / 2} \xi_{2}}^{(2)} \Pi .
\end{aligned}
$$

We now introduce into the master Eq. (8) the birth and death rates, which were defined in Section 2. Thus, the first two terms on the right-hand-side (RHS) of (8) become

$$
\left(-\Omega^{-1 / 2} \frac{\partial}{\partial \xi_{1}}+\frac{1}{2} \Omega^{-1} \frac{\partial^{2}}{\partial \xi_{1}^{2}}\right) \alpha_{\Omega x_{1}+\Omega^{1 / 2} \xi_{1}, \Omega x_{2}+\Omega^{1 / 2} \xi_{2}} \Pi
$$




$$
\begin{aligned}
& +\left(\Omega^{-1 / 2} \frac{\partial}{\partial \xi_{1}}+\frac{1}{2} \Omega^{-1} \frac{\partial^{2}}{\partial \xi_{1}^{2}}\right) \beta_{\Omega x_{1}+\Omega^{1 / 2} \xi_{1}, \Omega x_{2}+\Omega^{1 / 2} \xi_{2}} \Pi=\left(-\alpha x_{1} \Omega^{1 / 2}\right) \frac{\partial \Pi}{\partial \xi_{1}} \\
& +\left(\frac{\alpha}{2} x_{1} \frac{\partial^{2} \Pi}{\partial \xi_{1}^{2}}-\alpha \frac{\partial\left(\xi_{1} \Pi\right)}{\partial \xi_{1}}\right)+\left(\beta x_{1} \Omega^{1 / 2}\right) \frac{\partial \Pi}{\partial \xi_{1}}+\left(\frac{\beta}{2} x_{1} \frac{\partial^{2} \Pi}{\partial \xi_{1}^{2}}+\beta \frac{\partial\left(\xi_{1} \Pi\right)}{\partial \xi_{1}}\right) \\
& +O\left(\Omega^{-1 / 2}\right) .
\end{aligned}
$$

Notice that parameters $\bar{\mu}^{(1)}, \bar{\mu}^{(2)}$ and $\bar{\gamma}$ are the rate of interactions between tumor-normal or tumor-tumor cells and, therefore, depend on $\Omega$. Thus, it is assumed that $\bar{\mu}^{(1)}=\mu^{(1)} / \Omega, \bar{\mu}^{(2)}=\mu^{(2)} / \Omega$ and $\bar{\gamma}=\gamma / \Omega$, for $\mu^{(1)}, \mu^{(2)}, \gamma>0$. The third term on the RHS of (8) can then be written as

$$
\begin{aligned}
& \left(\Omega^{-1 / 2} \frac{\partial}{\partial \xi_{1}}+\frac{1}{2} \Omega^{-1} \frac{\partial^{2}}{\partial \xi_{1}^{2}}\right) \gamma_{\Omega x_{1}+\Omega^{1 / 2} \xi_{1}, \Omega x_{2}+\Omega^{1 / 2} \xi_{2}} \Pi \\
& =\left(\gamma \Omega^{1 / 2} x_{1}^{2}\right) \frac{\partial \Pi}{\partial \xi_{1}}+\gamma\left(2 x_{1} \frac{\partial\left(\xi_{1} \Pi\right)}{\partial \xi_{1}}+\frac{1}{2} x_{1}^{2} \frac{\partial^{2} \Pi}{\partial \xi_{1}^{2}}\right)+O\left(\Omega^{-1 / 2}\right),
\end{aligned}
$$

and the fourth term of the forward Kolmogorov Eq. (8) becomes

$$
\begin{aligned}
& \left(\Omega^{-1 / 2} \frac{\partial}{\partial \xi_{1}}+\frac{1}{2} \Omega^{-1} \frac{\partial^{2}}{\partial \xi_{1}^{2}}\right) \mu_{\Omega x_{1}+\Omega^{1 / 2} \xi_{1}, \Omega x_{2}+\Omega^{1 / 2} \xi_{1}}^{(1)} \Pi \\
& =\left(\mu^{(1)} \Omega^{1 / 2} x_{1} x_{2}\right) \frac{\partial \Pi}{\partial \xi_{1}}+\left(\frac{\mu^{(1)}}{2} x_{1} x_{2} \frac{\partial^{2} \Pi}{\partial \xi_{1}^{2}}+\mu^{(1)} x_{1} \frac{\partial\left(\xi_{2} \Pi\right)}{\partial \xi_{1}}+\mu^{(1)} x_{2} \frac{\partial\left(\xi_{1} \Pi\right)}{\partial \xi_{1}}\right)+O\left(\Omega^{-1 / 2}\right) .
\end{aligned}
$$

For the fifth term of (8), we have, from Section 2,

$$
\mu_{\Omega x_{1}+\Omega^{\frac{1}{2}} \xi_{1}, \Omega x_{2}+\Omega^{\frac{1}{2} \xi_{2}}}^{(2)}=\bar{\mu}^{(2)} g\left(\Omega x_{1}+\Omega^{\frac{1}{2}} \xi_{1}, \Omega x_{2}+\Omega^{\frac{1}{2}} \xi_{2}\right) .
$$

Using the fact that $g$ is an homogeneous function of order two, the above expression becomes

$$
\mu_{\Omega x_{1}+\Omega^{\frac{1}{2}} \xi_{1}, \Omega x_{2}+\Omega^{\frac{1}{2}} \xi_{2}}^{(2)}=\bar{\mu}^{(2)} \Omega^{2} g\left(x_{1}+\frac{1}{\Omega^{\frac{1}{2}}} \xi_{1}, x_{2}+\frac{1}{\Omega^{\frac{1}{2}}} \xi_{2}\right) .
$$

Then, expanding $g$ into a Taylor series around $\left(x_{1}, x_{2}\right)$ we arrive at

$$
\mu_{\Omega x_{1}+\Omega^{\frac{1}{2}} \xi_{1}, \Omega x_{2}+\Omega^{\frac{1}{2}} \xi_{2}}^{(2)}=g\left(x_{1}, x_{2}\right)+\frac{1}{\Omega^{\frac{1}{2}}}\left(\frac{\partial g\left(x_{1}, x_{2}\right)}{\partial x_{1}} \xi_{1}+\frac{\partial g\left(x_{1}, x_{2}\right)}{\partial x_{2}} \xi_{2}\right)+O\left(\Omega^{-1}\right) .
$$

Introducing this last equation in the fifth term of Eq. (8), we get

$$
\begin{aligned}
& \left(\Omega^{-1 / 2} \frac{\partial}{\partial \xi_{2}}+\frac{1}{2} \Omega^{-1} \frac{\partial^{2}}{\partial \xi_{2}^{2}}\right) \mu_{\Omega x_{1}+\Omega^{1 / 2} \xi_{1}, \Omega x_{2}+\Omega^{1 / 2} \xi_{2}}^{(2)} \Pi \\
& =\left(\mu^{(2)} \Omega^{\frac{1}{2}} g\left(x_{1}, x_{2}\right)\right) \frac{\partial \Pi}{\partial \xi_{2}}+\left(\frac{\mu^{(2)}}{2} g\left(x_{1}, x_{2}\right) \frac{\partial^{2} \Pi}{\partial \xi_{2}^{2}}+\mu^{(2)} \frac{\partial g\left(x_{1}, x_{2}\right)}{\partial x_{1}} \frac{\partial\left(\xi_{1} \Pi\right)}{\partial \xi_{2}}+\mu^{(2)} \frac{\partial g\left(x_{1}, x_{2}\right)}{\partial x_{2}} \frac{\partial\left(\xi_{2} \Pi\right)}{\partial \xi_{2}}\right)+O\left(\Omega^{-1 / 2}\right) .
\end{aligned}
$$

Collecting Eqs. (9)-(12) of Eq. (8), we arrive at the following equation

$$
\begin{aligned}
\frac{\partial \Pi}{\partial t}- & \Omega^{\frac{1}{2}} \frac{d x_{1}}{d t} \frac{\partial \Pi}{\partial \xi_{1}}-\Omega^{\frac{1}{2}} \frac{d x_{2}}{d t} \frac{\partial \Pi}{\partial \xi_{2}}=\Omega^{\frac{1}{2}}\left(\left(-\alpha x_{1}+\beta x_{1}+\gamma x_{1}^{2}+\mu^{(1)} x_{1} x_{2}\right) \frac{\partial \Pi}{\partial \xi_{1}}+\mu^{(2)} g\left(x_{1}, x_{2}\right) \frac{\partial \Pi}{\partial \xi_{2}}\right) \\
& +\Omega^{0}\left(\left(\frac{\alpha+\beta+\gamma x_{1}+\mu^{(1)} x_{2}}{2}\right) x_{1} \frac{\partial^{2} \Pi}{\partial \xi_{1}^{2}}+\left(\beta-\alpha+2 \gamma x_{1} \mu^{(1)} x_{2}\right) \frac{\partial\left(\xi_{1} \Pi\right)}{\partial \xi_{1}}+\mu^{(1)} x_{1} \frac{\partial\left(\xi_{2} \Pi\right)}{\partial \xi_{1}}\right. \\
& \left.+\frac{\mu^{(2)}}{2} g\left(x_{1}, x_{2}\right) \frac{\partial^{2} \Pi}{\partial \xi_{2}^{2}}+\mu^{(2)} \frac{\partial g\left(x_{1}, x_{2}\right)}{\partial x_{2}} \frac{\partial\left(\xi_{2} \Pi\right)}{\partial \xi_{2}}+\mu^{(2)} \frac{\partial g\left(x_{1}, x_{2}\right)}{\partial x_{1}} \frac{\partial\left(\xi_{1} \Pi\right)}{\partial \xi_{2}}\right)+O\left(\Omega^{-1 / 2}\right) .
\end{aligned}
$$

Since we are assuming that the number of cells is large enough, the deterministic process is considered as an approximation to the stochastic model. Gathering in (13) the terms of order $\Omega^{\frac{1}{2}}$ and since these terms are all either proportional to $\partial \Pi / \partial \xi_{1}$ or to $\partial \Pi / \partial \xi_{2}$ we arrive at the autonomous system

$$
\begin{aligned}
& \frac{d x_{1}(t)}{d t}=x_{1}\left(\alpha-\gamma x_{1}-\mu^{(1)} x_{2}\right)-\beta x_{1}, \\
& \frac{d x_{2}(t)}{d t}=-\mu^{(2)} g\left(x_{1}, x_{2}\right),
\end{aligned}
$$


which are the equations for the deterministic densities. The first term in the RHS of Eq. (14) is the usual logistic form describing the effect of population saturation due to a maximum cell density. Likewise, $\alpha$ is the proliferation rate while the constants $\gamma$ and $\mu^{(1)}$ measure the tumor-tumor and the tumor-normal cell competition, respectively. The last term in (14) describes the tumor cell death, which could be the result of the interaction with the immune system and the reaction of the oedema.

In contrast, Eq. (15), where the constant $\mu^{(2)}>0$, only displays a decay of the normal cell density due to interaction with the tumor cells. As mentioned previously, the details of this interaction may be very complicated and are embodied in the function $g$.

The terms of order $\Omega^{0}$ in (13) yield the Fokker-Planck equation

$$
\begin{aligned}
\frac{\partial \Pi}{\partial t}= & \left(\beta-\alpha+2 \gamma x_{1}+\mu^{(1)} x_{2}\right) \frac{\partial\left(\xi_{1} \Pi\right)}{\partial \xi_{1}}+\mu^{(1)} x_{1} \frac{\partial\left(\xi_{2} \Pi\right)}{\partial \xi_{1}}+\mu^{(2)} \frac{\partial g\left(x_{1}, x_{2}\right)}{\partial x_{1}} \frac{\partial\left(\xi_{1} \Pi\right)}{\partial \xi_{2}}+\mu^{(2)} \frac{\partial g\left(x_{1}, x_{2}\right)}{\partial x_{2}} \frac{\partial\left(\xi_{2} \Pi\right)}{\partial \xi_{2}} \\
& +\left(\frac{\alpha+\beta+\gamma x_{1}+\mu^{(1)} x_{2}}{2}\right) x_{1} \frac{\partial^{2} \Pi}{\partial \xi_{1}^{2}}+\frac{\mu^{(2)}}{2} g\left(x_{1}, x_{2}\right) \frac{\partial^{2} \Pi}{\partial \xi_{2}^{2}}+O\left(\Omega^{-1 / 2}\right) .
\end{aligned}
$$

From (16), the equations for the moments of $\xi_{1}$ and $\xi_{2}$ can be derived and read as

$$
\begin{aligned}
& \frac{d\left\langle\xi_{1}\right\rangle}{d t}=-\left(\beta-\alpha+2 \gamma x_{1}+\mu^{(1)} x_{2}\right)\left\langle\xi_{1}\right\rangle-\mu^{(1)} x_{1}\left\langle\xi_{2}\right\rangle, \\
& \frac{d\left\langle\xi_{2}\right\rangle}{d t}=-\mu^{(2)} \frac{\partial g\left(x_{1}, x_{2}\right)}{\partial x_{1}}\left\langle\xi_{1}\right\rangle-\mu^{(2)} \frac{\partial g\left(x_{1}, x_{2}\right)}{\partial x_{2}}\left\langle\xi_{2}\right\rangle .
\end{aligned}
$$

Moreover, the three second-order moments obey the three coupled equations:

$$
\begin{aligned}
& \frac{d\left\langle\xi_{1}^{2}\right\rangle}{d t}=-2\left(\beta-\alpha+2 \gamma x_{1}+\mu^{(1)} x_{2}\right)\left\langle\xi_{1}^{2}\right\rangle-2 \mu^{(1)} x_{1}\left\langle\xi_{1} \xi_{2}\right\rangle+\left(\alpha+\beta+\gamma x_{1}+\mu^{(1)} x_{2}\right) x_{1}, \\
& \frac{d\left\langle\xi_{1} \xi_{2}\right\rangle}{d t}=-\left(\beta-\alpha+2 \gamma x_{1}+\mu^{(1)} x_{2}+\mu^{(2)} \frac{\partial g\left(x_{1}, x_{2}\right)}{\partial x_{2}}\right)\left\langle\xi_{1} \xi_{2}\right\rangle-\mu^{(2)} \frac{\partial g\left(x_{1}, x_{2}\right)}{\partial x_{1}}\left\langle\xi_{1}^{2}\right\rangle-\mu^{(1)} x_{1}\left\langle\xi_{2}^{2}\right\rangle, \\
& \frac{d\left\langle\xi_{2}^{2}\right\rangle}{d t}=-2 \mu^{(2)} \frac{\partial g\left(x_{1}, x_{2}\right)}{\partial x_{1}}\left\langle\xi_{1} \xi_{2}\right\rangle-2 \mu^{(2)} \frac{\partial g\left(x_{1}, x_{2}\right)}{\partial x_{2}}\left\langle\xi_{2}^{2}\right\rangle+\mu^{(2)} g\left(x_{1}, x_{2}\right) .
\end{aligned}
$$

\section{Mathematical analysis of the model}

\subsection{Existence of solutions, qualitative analysis and stability of the deterministic system}

In this subsection, a study of the existence of solutions of the deterministic system (14), (15) is considered. We first specify a set of additional properties to be satisfied by function $g\left(x_{1}, x_{2}\right)$ :

(H1) $g\left(x_{1}, x_{2}\right)=x_{1} x_{2} h\left(x_{1}, x_{2}\right)$, with $h \in \mathcal{C}^{1}\left(\mathbb{R}^{2}\right)$.

(H2) $h\left(x_{1}, x_{2}\right)>0, \forall x_{1}, x_{2}>0$.

(H3) $\frac{\partial h\left(x_{1}, x_{2}\right)}{\partial x_{1}} \geq 0, \forall x_{1}, x_{2} \geq 0$, except possibly in a set of zero measure.

We state the following result:

Theorem 1. For any initial data $\left(x_{10}, x_{20}\right)$ such that $x_{10}>0$ and $x_{20}>0$ the solutions to the system (14) and (15) exist for $t>$ 0 , are positive bounded and unique.

Proof. Representing the solutions of system (14) and (15) in integral form:

$$
\begin{aligned}
& x_{1}(t)=x_{10} \exp \left[\int_{0}^{t}\left[\alpha-\beta-\gamma x_{1}(s)-\mu^{(1)} x_{2}(s)\right] d s\right], \\
& x_{2}(t)=x_{20} \exp \left[-\int_{0}^{t} \mu^{(2)} h\left(x_{1}(s)\right) d s\right] .
\end{aligned}
$$

Thus, if the initial conditions are positive, the components of the solution are positive for all finite time $t$.

Moreover, since $\alpha-\beta-\gamma x_{1}(s)-\mu^{(1)} x_{2}(s) \geq 0, \forall s>0$, then

$$
\frac{d x_{1}}{d t} \leq x_{1}\left(\alpha-\beta-\gamma x_{1}\right) \text {. }
$$

This differential inequality shows that $x_{1}(t)$ is bounded. The boundedness of $x_{2}$ is straightforward from (15) as it is a decreasing function. Given that the RHS of Eqs. (14) and (15) are continuous in $\left(x_{1}, x_{2}\right)$ and have bounded coefficients, the 
existence of solutions follows from [44]. Furthermore, as the partial derivatives of the RHS of the system are continuous and bounded, uniqueness follows from the Picard-Lindelof theorem.

Next, we rule out the presence of limit cycles on account of the Bendixon-Dulac theorem. Let $Q$ be the first quadrant of the phase plane $\left(x_{1}, x_{2}\right)$,

$$
Q=\left\{\left(x_{1}, x_{2}\right): x_{1}>0, x_{2}>0\right\} \text {. }
$$

Then, we formulate the following theorem:

Theorem 2. If conditions (H1)-(H3) are satisfied then, there exist no limit cycles of the system (14), (15) in the region $Q$.

Proof. Let us define an auxiliary function $B\left(x_{1}, x_{2}\right)=\frac{1}{g\left(x_{1}, x_{2}\right)}$. The (Dulac) function $B$ is continuously differentiable on the simply connected region $Q$. Using now the Bendixon-Dulac theorem we check the sign of

$$
\frac{\partial}{\partial x_{1}}\left[B\left(x_{1}, x_{2}\right) x_{1}\left(\alpha-\beta-\gamma x_{1}-\mu^{(1)} x_{2}\right)\right]+\frac{\partial}{\partial x_{2}}\left[B\left(x_{1}, x_{2}\right)\left(-\mu^{(2)} g\left(x_{1}, x_{2}\right)\right)\right] \text {. }
$$

Since $g\left(x_{1}, x_{2}\right)=x_{1} x_{2} h\left(x_{1}, x_{2}\right)$, the above expression reduces to

$$
\frac{\partial}{\partial x_{1}}\left[\frac{x_{1}\left(\alpha-\beta-\gamma x_{1}-\mu^{(1)} x_{2}\right)}{g\left(x_{1}, x_{2}\right)}\right]=\frac{-\left(\alpha-\beta-\gamma x_{1}-\mu^{(1)} x_{2}\right) \frac{\partial h}{\partial x_{1}}-\gamma h}{x_{2} h^{2}}<0,
$$

for all $\left(x_{1}, x_{2}\right) \in Q$. Therefore, due to the Bendixon-Dulac theorem, no periodic solutions exist for the system (14), (15) lying entirely within the region $Q$.

Now, we perform an asymptotic analysis for the system of Eqs. (14) and (15). The equilibrium points of the system are then, using (H1) and (H2)

$$
P_{1}=\left(\frac{\alpha-\beta}{\gamma}, 0\right), \quad P_{2}=(0, a), \quad a \geq 0 .
$$

Since $\alpha$ and $\beta$ represent the birth and death rates of the tumor cells, unless there exists an exogenous factor (such as therapy) altering these rates, we assume $\alpha>\beta$. Thus, the previous equilibrium points are non-negative and they remain inside the first quadrant.

In order to check the stability of the fixed points, the Jacobian matrix $J$ is calculated for system (14), (15).

$$
J\left(x_{1}, x_{2}\right)=\left(\begin{array}{cc}
\alpha-2 \gamma x_{1}-\mu^{(1)} x_{2}-\beta & -\mu^{(1)} x_{1} \\
-\mu^{(2)} \frac{\partial g\left(x_{1}, x_{2}\right)}{\partial x_{1}} & -\mu^{(2)} \frac{\partial g\left(x_{1}, x_{2}\right)}{\partial x_{2}}
\end{array}\right) .
$$

- Substituting the fixed point $P_{1}$ in the matrix, using hypothesis (H3) and calculating the eigenvalues, we obtain $\lambda_{1}=$ $-(\alpha-\beta)<0$ and $\lambda_{2}=-\mu^{(2)} \frac{\partial g\left(P_{1}\right)}{\partial x_{2}}<0$. Thus, $P_{1}$ is a stable node.

- Substituting $P_{2}$ in $J$, employing (H3) and calculating the eigenvalues, we get $\lambda_{1}=0$ and $\lambda_{2}=\alpha-\mu^{(1)} a-\beta$. These points are nonhyperbolic points. If $\mu^{(1)} a<\alpha-\beta$, then the fixed points $P_{2}$ possess a local unstable manifold and a local center manifold. Otherwise, $P_{2}$ has a local stable and a local center manifolds.

From a biological point of view, it is reasonable to concentrate on the situation where $a<\frac{\alpha-\beta}{\mu^{(1)}}$. In this case, $\lambda_{2}=\alpha-$ $\mu^{(1)} a-\beta>0$ and the fixed points $P_{2}$ are all unstable. Therefore, this constraint will be considered henceforth, and, thus, we rule out the points for which $a>\frac{\alpha-\beta}{\mu^{(1)}}$.

Now, we analyze the behavior of the nullclines of the system (14), (15).

- Nullcline for $x_{1}$. The nullclines for (14) are

$$
x_{1}=0, \quad x_{2}=\frac{\alpha-\beta}{\mu^{(1)}}-\frac{\gamma}{\mu^{(1)}} x_{1} .
$$

- Nullcline for $x_{2}$. From (H1), we have that for Eq. (15), the nullclines are $x_{1}=0$ and $x_{2}=0$.

Therefore, we can formulate the following proposition:

Proposition 1. If the assumptions $(\mathrm{H} 1)-(\mathrm{H} 3)$ are fulfilled, then the steady state $P_{1}=\left(\frac{\alpha-\beta}{\gamma}, 0\right)$ is globally stable in the following region $D$ :

$$
0 \leq x_{1} \leq \frac{\alpha-\beta}{\gamma}, \quad 0 \leq x_{2} \leq \frac{\alpha-\beta}{\mu^{(1)}}-\frac{\gamma}{\mu^{(1)}} x_{1} .
$$

Proof. Let us consider the triangular region $D$ defined in (25). Denote by $\vec{n}$ the outward-pointing normal vector for each segment of the boundary $D$. The flow on each segment of the region $D$ is given by the scalar product:

$$
\left.X\right|_{\partial D}=\left.\left\langle\vec{n},\left(x_{1}\left(\alpha-\gamma x_{1}-\mu^{(1)} x_{2}\right)-\beta x_{1},-\mu^{(2)} g\left(x_{1}, x_{2}\right)\right)\right\rangle\right|_{\partial D} .
$$



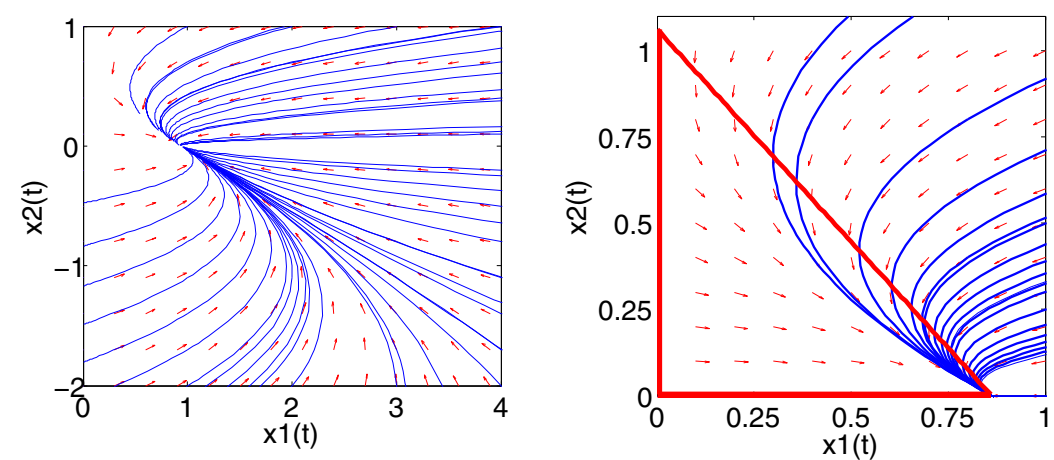

Fig. 1. (Left panel) Sketch of the phase portrait for Eqs. (14) and (15) in the case when $g\left(x_{1}, x_{2}\right)=x_{1} x_{2}$ and for values of the parameters given in Table 1 . (Right panel) Zoom of the left panel plot to display the positive invariant region $D$ (red triangle). (For interpretation of the references to colour in this figure legend, the reader is referred to the web version of this article.)

Table 1

Values of the biological parameters for the system (14), (15) extracted from $[9,12,29,51]$.

\begin{tabular}{ll}
\hline Variable & Value (Units) \\
\hline$x_{10}$ & 0.2 \\
$x_{20}$ & 0.7 \\
$\alpha$ & 1.5 year $^{-1}$ \\
$\beta$ & 0.12 year $^{-1}$ \\
$\gamma$ & 1.6 year $^{-1}$ \\
$\mu^{(1)}$ & 1.3 year $^{-1}$ \\
$\mu^{(2)}$ & 0.8 year $^{-1}$ \\
\hline
\end{tabular}

- On the segment $r_{1}: x_{2}=\frac{\alpha-\beta}{\mu^{(1)}}-\frac{\gamma}{\mu^{(1)}} x_{1}$. It is straightforward to obtain $\left.X\right|_{r_{1}}=-\mu^{(2)} g\left(x_{1}, \frac{\alpha-\beta}{\mu^{(1)}}-\frac{\gamma}{\mu^{(1)}} x_{1}\right)<0$.

- On the segment $r_{2}: x_{1}=0$, we get $\left.X\right|_{r_{2}}=0$.

- Finally, on the segment $r_{3}: x_{2}=0$, we find that $\left.X\right|_{r_{3}}=\mu^{(2)} g\left(x_{1}, 0\right)=0$.

Then, $D$ is a positive invariant set of (14), (15). Therefore, all trajectories which attain $D$ remain there (see Fig. 1). Theorem 2 shows that there exist no limit cycles of Eqs. (14) and (15) in the region $D$. Therefore, the Poincare-Bendixon theorem implies that $P_{1}$ is globally asymptotically stable in $D$.

Fig. 1 depicts the phase portrait (left-hand panel) and the positive invariant region $D$ (right-hand panel) of Eqs. (14) and (15) for $g\left(x_{1}, x_{2}\right)=x_{1} x_{2}$ and the parameters displayed in Table 1.

Remark 1. There exists a very small probability for a fluctuation to drive the system, starting from $\left(x_{10}, x_{20}\right)$, backwards to extinction and therefore, discrepancy between the stochastic and deterministic equation could arise. The probability for this scenario to take place is typically of order $e^{-\Omega}$, and therefore, usually very small, except near the boundary of the attraction region. Although rare, the spontaneous regression of LGG has been documented in pediatric gliomas, either in association with neurofibromatosis, which increases the probability, or in its absence [57]. Despite the simplicity of our model, it is nevertheless worth mentioning that it can also account for this type of clinically observable process if suitable initial conditions $\left(x_{10}, x_{20}\right)$ are imposed. In what follows, we will however choose the initial conditions sufficiently far away from the $y$-axis and thus, the occurrence of such spontaneous regression will be negligible.

\subsection{The linear noise approximation: first and second moments of the fluctuations and their behavior near the stationary state}

Having analyzed the terms of order $\Omega^{1 / 2}$ in the previous section, we are left with an equation for $\Pi(\xi, t)$. The terms of order $\Omega^{0}$ were calculated in (16). This approximation is called linear noise approximation.

We now proceed to consider the behavior of this approximation, calculating the expected value, the variation and covariance of the fluctuations near the stationary steady states of the deterministic system, given by Eqs. (14) and (15). Thus, for $P_{1}=\left(\bar{x}_{1}, 0\right)=((\alpha-\beta) / \gamma, 0)$, the first two equations of (17) and (18) read as

$$
\begin{aligned}
& \frac{d\left\langle\xi_{1}\right\rangle}{d t}=-(\alpha-\beta)\left\langle\xi_{1}\right\rangle-\mu^{(1)} \frac{\alpha-\beta}{\gamma}\left\langle\xi_{2}\right\rangle, \\
& \frac{d\left\langle\xi_{2}\right\rangle}{d t}=-\mu^{(2)} \frac{\partial g\left(\bar{x}_{1}, 0\right)}{\partial x_{2}}\left\langle\xi_{2}\right\rangle .
\end{aligned}
$$



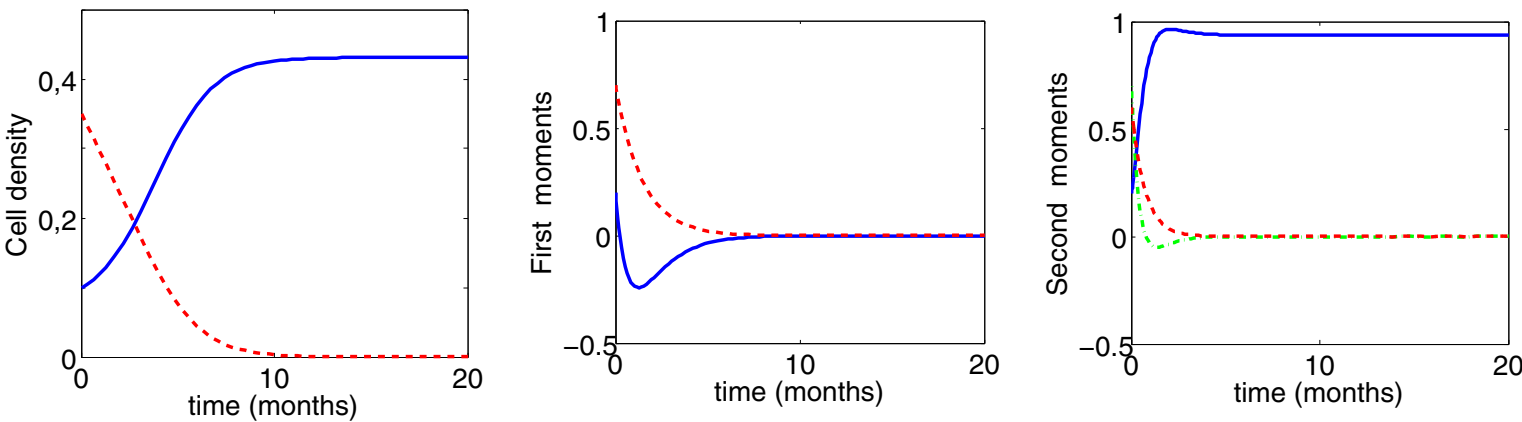

Fig. 2. (Left panel) The solution to Eqs. (14) and (15) (Middle panel) to Eqs. (27) (Right panel) and to Eqs. (28) for $g\left(x_{1}, x_{2}\right)=x_{1} x_{2}$ and for the parameters given in Table 1 . Blue and red colors label the curves for both populations, whereas the green color represents the curves for the moment $\left\langle\xi_{1} \xi_{2}\right\rangle$ (right panel). (For interpretation of the references to colour in this figure legend, the reader is referred to the web version of this article.)

It is straightforward to see that the trivial steady state $(0,0)$ of system $(27)$ is asymptotically stable. The system for the second-order moments becomes

$$
\begin{aligned}
\frac{d}{d t}\left\langle\xi_{1}^{2}\right\rangle & =-2(\alpha-\beta)\left\langle\xi_{1}^{2}\right\rangle-2 \mu^{(1)} \frac{\alpha-\beta}{\gamma}\left\langle\xi_{1} \xi_{2}\right\rangle+2 \alpha\left(\frac{\alpha-\beta}{\gamma}\right), \\
\frac{d}{d t}\left\langle\xi_{1} \xi_{2}\right\rangle & =-\left(\alpha-\beta+\mu^{(2)} \frac{\partial g\left(\bar{x}_{1}, 0\right)}{\partial x_{2}}\right)\left\langle\xi_{1} \xi_{2}\right\rangle-\mu^{(1)} \frac{\alpha-\beta}{\gamma}\left\langle\xi_{2}^{2}\right\rangle, \\
\frac{d}{d t}\left\langle\xi_{2}^{2}\right\rangle & =-2 \mu^{(2)} \frac{\partial g\left(\bar{x}_{1}, 0\right)}{\partial x_{2}}\left\langle\xi_{2}^{2}\right\rangle .
\end{aligned}
$$

It is easy to see that the steady state of system $(28)$ is $(\alpha / \gamma, 0,0)$ and a standard stability analysis shows that this steady state is asymptotically stable.

In Fig. 2, the solution to the deterministic system (14) and (15), expectations of fluctuations and the second moments, are shown. For these simulations, we have chosen a contact function $g\left(x_{1}, x_{2}\right)=x_{1} x_{2}$ and the parameters given in Table 1 . Notice that the time scale is in years since LGG progress very slowly in contrast with other higher-grade gliomas.

\section{Optimal control for the deterministic system of differential equations}

In standard chemotherapy protocols, drugs are administered in cycles at maximum tolerated doses (MTD) alternating with periods of rest. The underlying rationale is that the more anti-cancer drug is given to the patient, the more tumor cells are killed. Hence, the chemotherapeutic schedule traditionally used in the clinical practice consists of delivering the maximal dose of a chemotherapeutic (or a combination) that can be tolerated by the patient. However, as chemotherapy not only targets cancer cells but also any other cell in a state of rapid proliferation, the approach of MTD induces toxicities, depending on the type of anti-cancer agent used, and thus requires prolonged breaks between successive cycles to allow recovery from the harmful side effects. This eventually allows for (subsets of) malignant cells to resume their growth and, in the long term, the MTD efficacy can be hampered by the propensity of some cancer cells to develop resistance to the drugs.

There is a growing number of clinical evidences pointing to the fact that "more is not necessary better", which has led to the concept of biologically optimal dose (BOD) chemotherapy. An example is the so-called metronomic chemotherapy [7,37,52], i.e., the administration of traditional chemotherapy at lower, less toxic doses for longer times (even continuously). This idea has been put forward as an alternative on the basis of a growing experimental evidence not only because of its toxic effect on the tumor, but also because of the immune-stimulatory effects. By relying on lower doses and more frequent administration, metronomic chemotherapy can reduce toxic effects and prevent vascular rebound, a rapid vascular regrowth that can lead to tumor growth during therapy breaks [7,17]. The introduction of metronomic chemotherapy paved the way for the development of well-tolerated treatments that might prevent tumour progression for an extend period of time $[8,28]$. Initially, metronomic chemotherapy was thought to exert its antitumour activity exclusively though antiangiogenic mechanisms [33]. However, additional mechanisms of action include a enhanced immune response and additional anticancer effects, so metronomic chemotherapy is now considered to be a form of multi-targeted chemotherapy [52].

From the mathematical point of view, the modelling of cancer chemotherapy by using optimal control theory has various decades of history. Different frameworks have been considered in the literature, depending on the study focus, from macroscopic [60] to microscopic [37,38] level, including systems of differential equations (see $[20,25,55]$ and references therein) and partial differential equations (see instance [60] and references therein).

Here we formulate an optimal control problem for the administration of chemotherapy and particularize our results to the case of LGG, where two different modalities (time durations) are considered. We remind that since the median overall 
survival of LGG (WHO grade II) is about 7 years, these administration modalities extend for periods of many months (33 and 66 months).

\subsection{Formulation of the optimal control problem}

In this subsection, the following optimal control problem is studied

$$
\begin{aligned}
& \frac{d x_{1}}{d t}=\alpha x_{1}-\gamma x_{1}^{2}-\mu^{(1)} x_{1} x_{2}-\beta x_{1}-m_{1} u x_{1}, \quad x_{1}(0)=x_{10}, \\
& \frac{d x_{2}}{d t}=-\mu^{(2)} x_{1} x_{2}-m_{2} u x_{2}, \quad x_{2}(0)=x_{20},
\end{aligned}
$$

where the constants $m_{1}$ and $m_{2}$ are the characteristic rates of cell-death induced by chemotherapy in tumor and normal cells, respectively. Since we assume that the administered drug is cytotoxic, this implies that it will primarily (but not exclusively) interfere with the cell cycle of fast-dividing cells. Given that for LGG the proliferation rate is in the order of several months (see Table 1), the effect of chemotherapy is expected to produce visible effects within a period of few years. This is in stark contrast with high-grade gliomas (e.g., glioblastoma [5,45,53]) which exhibit a much faster dynamics (about two orders of magnitude). In our calculations, we will use the following values $m_{1}=2.0$ year $^{-1}$ and $_{2}=0.4$ year $^{-1}$.

The control variable $u$ represents the drug dose. In general, the main objective is to determine the optimal drug schedule which minimizes, as much as possible, the number of tumor cells at the final time of the treatment period while keeping the toxic side effects of the drug at an acceptable level. To reduce the toxicity of the drug, which also affects the normal cells, we add isoperimetric or state constraints. There are different kinds of constraints depending on the model assumptions, mainly pointwise or integral type constraints. For instance, one may prescribe a maximum rate at which the chemotherapeutic drug is administered at each time or imposes a bound on the total drug dosage in the therapy interval; cf. [20,21,39].

The set of admissible controls for the dynamical system (29) is given by

$$
U=\left\{u:[0, T] \rightarrow \mathbb{R} \text { measurable } \mid 0 \leq u(t) \leq u_{M} \text { for almost all } t \in[0, T]\right\} .
$$

Here, $u_{M}$, represents the MTD, which cannot be exceeded during the course of the therapy. In addition, we introduce another control constraint by imposing a bound on the total amount of drug dose administered:

$$
\int_{0}^{T} u(t) d t \leq M
$$

From a clinical point of view, the objective function should be designed in such a way that the tumor cell population $x_{1}$ is minimized by an admissible control $u$ satisfying the dynamical system (29) and the constraint (31). This goal can be achieved by minimizing the objective function

$$
J_{w}(u)=(1-w) x_{1}(T)+w \int_{0}^{T} x_{1}(t) d t
$$

where $w$ is a weight parameter. For $w=0$ we obtain a functional which focuses on the number of terminal tumor cells:

$$
J_{0}(u)=x_{1}(T)
$$

while for $w=1$ we get a functional which takes into account the dynamics of tumor cells during the entire therapy interval:

$$
J_{1}(u)=\int_{0}^{T} x_{1}(t) d t .
$$

By introducing the additional equation $\dot{z}=u(t), z(0)=0$, the constraint (31) is equivalent to the terminal constraint $z(T) \leq$ $M$. Then the control system can be written as:

$$
\begin{aligned}
& \frac{d x_{1}}{d t}=\alpha x_{1}-\gamma x_{1}^{2}-\mu^{(1)} x_{1} x_{2}-\beta x_{1}-m_{1} u x_{1}, \quad x_{1}(0)=x_{10}, \\
& \frac{d x_{2}}{d t}=-\mu^{(2)} x_{1} x_{2}-m_{2} u x_{2}, \quad x_{2}(0)=x_{20}, \\
& \frac{d z}{d t}=u, \quad z(0)=0, \quad z(T) \leq M .
\end{aligned}
$$

Hence, the state vector of the control problem is

$$
x=\left(x_{1}, x_{2}, z\right)^{\mathrm{T}} \in \mathbb{R}^{3} .
$$

Since the control variable $u$ appears linearly in the dynamics, we can write the model Eq. (35) in the form

$$
\dot{x}=f(x)+g(x) u,
$$


where

$$
f(x)=\left(\begin{array}{c}
\alpha x_{1}-\gamma x_{1}^{2}-\mu^{(1)} x_{1} x_{2}-\beta x_{1} \\
-\mu^{(2)} x_{1} x_{2} \\
0
\end{array}\right), \quad g(x)=\left(\begin{array}{c}
-m_{1} x_{1} \\
-m_{2} x_{2} \\
1
\end{array}\right) .
$$

\subsection{Pontryagin's Minimum Principle: necessary optimality conditions}

We evaluate the necessary optimality conditions of Pontryagin's Minimum Principle [26,40,54,59] for the optimal control problem with objective $J_{w}(u)$ in (32). Defining the adjoint variable $\lambda=\left(\lambda_{1}, \lambda_{2}, \lambda_{3}\right)$, the Hamiltonian is

$$
\begin{aligned}
H(x, \lambda, u)= & w x_{1}+\langle\lambda, f(x)+g(x) u\rangle \\
= & w x_{1}+\lambda_{1}\left(\alpha x_{1}-\gamma x_{1}^{2}-\mu^{(1)} x_{1} x_{2}-\beta x_{1}-m_{1} x_{1} u\right) \\
& +\lambda_{2}\left(-\mu^{(2)} x_{1} x_{2}-m_{2} x_{2} u\right)+\lambda_{3} u .
\end{aligned}
$$

The adjoint equation then becomes

$$
\dot{\lambda}=-H_{x}(x, \lambda, u)=-a_{1}-\lambda\left(D f(x)+u^{*} D g(x)\right), \quad \lambda(T)=m,
$$

where $a_{1}=(w, 0,0)^{t}, m=(1-w, 0)^{t}$ and $D f$, resp., $D g$ denote the matrices of the partial derivatives of the vector fields $f$, resp., g.

The optimal control $u^{*}$ minimizes the Hamiltonian $H$ which is equivalent to minimizing the function

$$
\left[-m_{1} x_{1} \lambda_{1}-m_{2} x_{2} \lambda_{2}+\lambda_{3}\right] u,
$$

with respect to $u \in\left[0, u_{M}\right]$. Hence, defining the switching function $\phi(x, \lambda)$ as

$$
\phi(x, \lambda)=-m_{1} x_{1} \lambda_{1}-m_{2} x_{2} \lambda_{2}+\lambda_{3},
$$

and setting

$$
\phi(t)=\phi(x(t), \lambda(t))
$$

the optimal control satisfies the switching condition

$$
u^{*}(t)= \begin{cases}0, & \text { if } \phi(t)>0 \\ u_{M}, & \text { if } \phi(t)<0\end{cases}
$$

In later computations, we shall take the MTD $u_{M}=1$. The control is not determined a priori by the minimum condition at times when $\phi(t)=0$. Thus, we consider two cases:

- If the switching $\phi(t)$ has only finitely many zeros on an interval $I_{b} \subset[0, T]$, the control $u^{*}$ is called bang-bang on $I_{b}$. In this case we have $u^{*}(t) \in\left\{0, u_{M}\right\}$ for all $t \in I_{b}$.

- If $\phi(t) \equiv 0$ holds on a time interval $I_{S}=\left[t_{1}, t_{2}\right], 0 \leq t_{1}<t_{2} \leq T$, we say $u^{*}$ is singular on $I_{s}$.

There exists a wealth of literature analyzing bang-bang and singular controls and presenting numerical methods; cf $[11,14,46,59]$.

\subsection{Computation of singular controls}

The switching function (42) can be written in the form

$$
\phi(x, \lambda)=\langle\lambda, g(x)\rangle,
$$

where $\langle$,$\rangle denotes the inner product. Formulas for singular controls can be derived using the fact that all total time deriva-$ tives of the switching function vanish on the singular interval $I_{s}$. In the sequel, we shall use methods from Geometric Control Theory [59] to compute the derivatives of $\phi$. To this end, we resort to the notion of Lie brackets [59].

Definition 1. Let $f$ and $g$ be two continuously differentiable vector fields. Then the Lie bracket of $f$ and $g$ in local coordinates is defined by

$$
[f, g](x)=D g(x) f(x)-D f(x) g(x) .
$$

Now, in view of $\phi(x, \lambda)=\langle\lambda, g(x)\rangle$, we obtain the first two total time derivatives of the switching function as

$$
\begin{gathered}
\frac{d}{d t} \phi(x, \lambda)=-a_{1} g(x)+\lambda[f, g](x), \\
\frac{d^{2}}{d t^{2}} \phi(x, \lambda)=M(x, \lambda)+N(x, \lambda) u,
\end{gathered}
$$

where 


$$
\begin{aligned}
& M(x, \lambda)=\langle\lambda,[f,[f, g]](x)\rangle-a_{1}\langle\operatorname{Dg}(x), f(x)\rangle-\left\langle a_{1},[f, g](x)\right\rangle, \\
& N(x, \lambda)=\langle\lambda,[g,[f, g]](x)\rangle-a_{1}\langle\operatorname{Dg}(x), g(x)\rangle .
\end{aligned}
$$

It has been shown in Krener [34] that the control variable $u$ appears only in an even order derivative of the switching function. The order of a singular control $u$ on an interval $I_{s}=\left[t_{1}, t_{2}\right]$ is defined as the least integer $q$ such that

$$
\frac{\partial}{\partial u} \frac{d^{2 q}}{d t^{2 q}} \phi \neq 0
$$

In view of (46) the order of a singular control in our problem is $q=1$, since

$$
\frac{\partial}{\partial u} \frac{d^{2}}{d t^{2}} \phi=N(x, \lambda) \neq 0 .
$$

A further necessary optimality condition is the generalized Legendre-Clebsch condition (GLC); cf[11,14,34].:

$$
-\frac{\partial}{\partial u} \frac{d^{2}}{d t^{2}} \phi(t)=-N(x(t), \lambda(t)) \geq 0, \quad \forall t \in I_{s} .
$$

In order to determine the singular control $u$ from the representation (46) we assume that the strict GLC condition holds:

$$
-\frac{\partial}{\partial u} \frac{d^{2}}{d t^{2}} \phi(t)=-N(x(t), \lambda(t))>0, \quad \forall t \in I_{s}
$$

In our problem, the strict GLC condition cannot be verified a priori, but has to be checked numerically. Assuming now (51), the relation $\frac{d^{2}}{d t^{2}} \phi(t)=0$ yields the following formula of the singular control in terms of the state variable $x$ and adjoint variable $\lambda$ :

$$
u_{\text {sing }}(x(t), \lambda(t))=-\frac{M(x(t), \lambda(t))}{N(x(t), \lambda(t))} .
$$

Then the optimal control on the singular interval $I_{s}$ is given by

$$
u^{*}(t)=u_{\text {sing }}(x(t), \lambda(t)), \quad \forall t \in I_{s} .
$$

To obtain the functions $M$ and $N$ we compute the following Lie brackets:

$$
[f, g](x)=\left(\begin{array}{c}
-\gamma m_{1} x_{1}^{2}-\mu^{(1)} x_{1} m_{2} x_{2} \\
-\mu^{(2)} x_{2} m_{1} x_{1} \\
0
\end{array}\right)
$$

and

$$
[g,[f, g]](x)=\left(\begin{array}{c}
\gamma m_{1}^{2} x_{1}^{2}+\mu^{(1)} x_{1} m_{2}^{2} x_{2} \\
\mu^{(2)} x_{2} m_{1}^{2} x_{1} \\
0
\end{array}\right)
$$

and

$$
[f,[f, g]](x)=\left(\begin{array}{c}
\gamma m_{1}(\beta-\alpha) \alpha x_{1}^{2}+\mu^{(1)}\left(m_{1}-m_{2}\right)\left(\gamma-\mu^{(2)}\right) x_{1}^{2} x_{2} \\
\mu^{(2)} \mu^{(1)}\left(m_{1}-m_{2}\right) x_{1} x_{2}^{2}+\mu^{(2)} m_{1}(\beta-\alpha) x_{1} x_{2} \\
0
\end{array}\right) .
$$

This allows us to find the functions $M$ and $N$ in (47) and (48) explicitly:

$$
\begin{aligned}
& M(x(t), \lambda(t))=\lambda_{1} L_{1}+\lambda_{2} L_{2}+w x_{1}\left[m_{1}\left(\alpha-\mu^{(1)} x_{2}-\beta\right)+\mu^{(1)} m_{2} x_{2}\right], \\
& N(x(t), \lambda(t))=\lambda_{1}\left(\gamma m_{1}^{2} x_{1}^{2}+\mu^{(1)} m_{2}^{2} x_{1} x_{2}\right)+\lambda_{2} \mu^{(2)} m_{1}^{2} x_{1} x_{2}-w m_{1}^{2} x_{1},
\end{aligned}
$$

where

$$
\begin{aligned}
L_{1}= & -\gamma m_{1} \alpha x_{1}^{2}+\gamma m_{1} \mu^{(1)} x_{1}^{2} x_{2}+\gamma m_{1} \beta x_{1}^{2}-\gamma \mu^{(1)} m_{2} x_{1}^{2} x_{2} \\
& +\left(m_{2}-m_{1}\right) \mu^{(1)} \mu^{(2)} x_{1}^{2} x_{2}, \\
L_{2}= & -\mu^{(2)} m_{1} \alpha x_{1} x_{2}+\mu^{(2)} m_{1} \mu^{(1)} x_{1} x_{2}^{2}+\mu^{(2)} m_{1} \beta x_{1} x_{2}-\mu^{(2)} \mu^{(1)} m_{2} x_{1} x_{2}^{2} .
\end{aligned}
$$

The strict GLC condition (51) then requires to check whether the inequality

$$
-\lambda_{1}\left(\gamma m_{1}^{2} x_{1}^{2}+\mu^{(1)} m_{2}^{2} x_{1} x_{2}\right)-\lambda_{2}\left(\mu^{(2)} m_{1}^{2} x_{1} x_{2}\right)+w m_{1}^{2} x_{1}>0,
$$

holds on the singular interval $I_{s}$. 

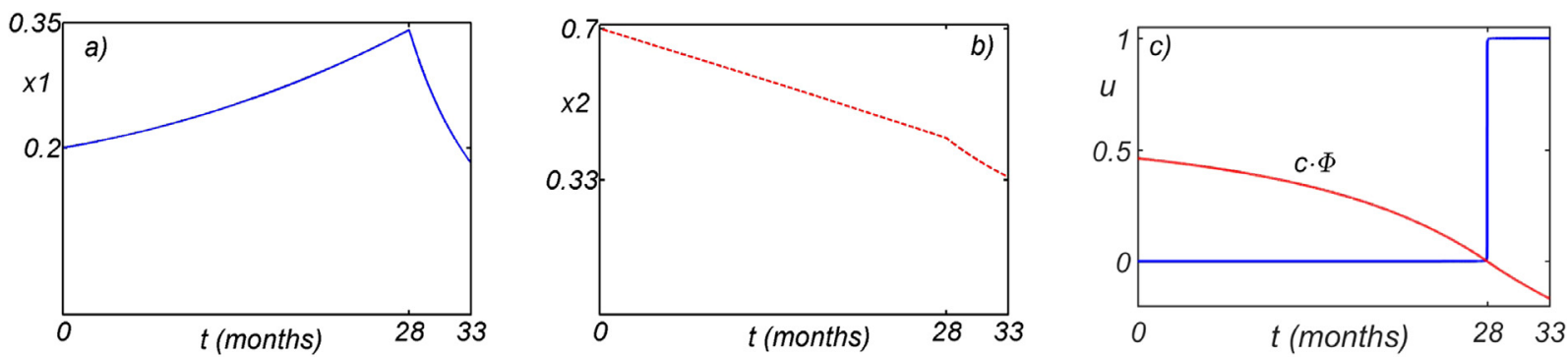

Fig. 3. Optimal solution for functional $J_{w}(u)$ in Eq. (59) with $w=0$ and total dose $M=5$; the time horizon is $T=33$ months. Left: a) Tumor cells $x_{1}$. Middle: b) Normal cells $x_{2}$. Right: c) Optimal control $u^{*}(t)$ and scaled switching function $c \cdot \phi$ with $c=30$, where $\phi$ satisfies the control law (43).

\subsection{Optimal control solutions}

\subsubsection{Sketch of numerical methods.}

Several numerical methods are available to solve the class of optimal control problem with constraints considered here. We choose the numerical approach First Discretize then Optimize. Using a suitable discretization scheme the optimal control problem is transformed into a large-scale nonlinear programming (NLP) problem; cf., e.g., [13,46,62]. The NLP problem can be efficiently solved by the Interior-Point code IPOPT developed by Wächter and Biegler [62]. Here, we formulate the discretized control problem with the help of the Applied Model-Interior-Point optimization code IPOPT. We use the implicit Euler method for ODEs. The Lagrange multipliers of the NLP problem represent the discretized adjoint variables of the original optimal control problem. This allows us to verify the necessary optimality conditions; in particular the switching condition (43) with high precision. Verifiable sufficient optimality conditions have been developed only for bang-bang controls; cf. $[46,50]$.

\subsubsection{Optimal controls for the functional $\mathrm{J}_{\mathrm{w}}(\mathrm{u})$ in (32)}

The goal of this section is to compute a standard protocol of chemotherapy, where the total dose of chemotherapy is $M=5$ to be administered during two different treatment modalities: $T=33$ and $T=66$ months, respectively. We use the parameters of Table 1.

We consider the weighted objective function (32):

$$
J_{w}(u)=(1-w) x_{1}(T)+w \int_{0}^{T} x_{1}(t) d t, \quad 0 \leq w \leq 1
$$

and, within each modality, several cases are distinguished:

Case $w=0, T=33$ months.

The state variables, the optimal control and the switching are shown in Fig. 3. Initially, no dose is applied during the first 28 months, and subsequently the MTD is administered for the last 5 months. It can be seen that tumor cells grow while normal cells are diminished during the no-treatment period. Once the chemotherapy starts, both types of cells decay with the tumor cells displaying a more pronounced decline.

We obtain a bang-bang optimal control given by ( $\mathrm{t}$ in months):

$$
u^{*}(t)= \begin{cases}0, & \text { for } 0 \leq t<t_{1}, \\ 1, & \text { for } t_{1} \leq t \leq T,\end{cases}
$$

and terminal states

$$
J_{0}\left(u^{*}\right)=0.181541, \quad x_{1}(T)=0.181541, \quad x_{2}(T)=0.336674 .
$$

From the simulations, we find $t_{1}=28$ months.

To show the optimality of the control $u^{*}(t)$ in Fig. 3c), we apply the second order sufficient condition (SSC) by Osmolovskii and Maurer (Theorem 7.10 in [50]). Since there is only one switching point $t_{1}$ and one terminal constraint $z(T)=5$, the SSC degenerates into a first-order sufficient condition, which is fulfilled if the following strict bang-bang property holds:

$$
\phi(t)>0, \quad \forall t \in\left[0, t_{1}\right], \quad \dot{\phi}\left(t_{1}\right)<0, \quad \phi(t)<0, \quad \forall t \in\left(t_{1}, T\right] .
$$

The dotted line in Fig. 3c) shows that the strict bang-bang property holds.

Case $w=1, T=33$ months.

The state variables, the optimal control and the switching are shown in Fig. 4. Now, in contrast to the solution for $w=0$, the maximal dose is administered from the very beginning until the total dose $M=5$ is reached. Thereafter, no treatment is administered. Both the tumor and normal cells decrease until the treatment finishes and the tumor cells regrow, while the normal cells are depleted although at a slower pace. 

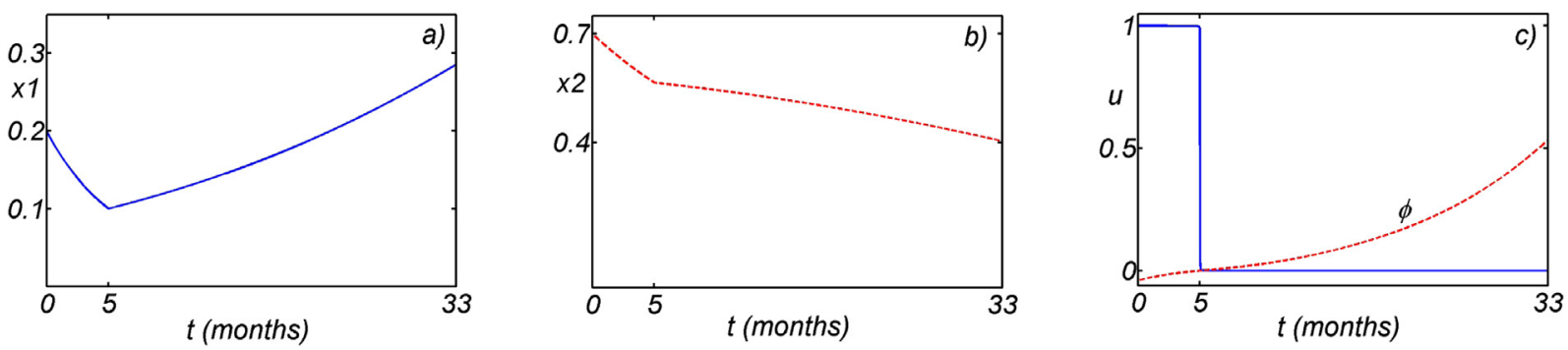

Fig. 4. Optimal solution for functional $J_{w}(u)$ in Eq. (59) with $w=1$, and total dose $M=5$; the time horizon is $T=33$ months. Left: a) Tumor cells $x_{1}$ Middle: b) Normal cells $x_{2}$; Right: c) Optimal control $u^{*}(t)$ and switching function $\phi$ satisfying the control law (43).
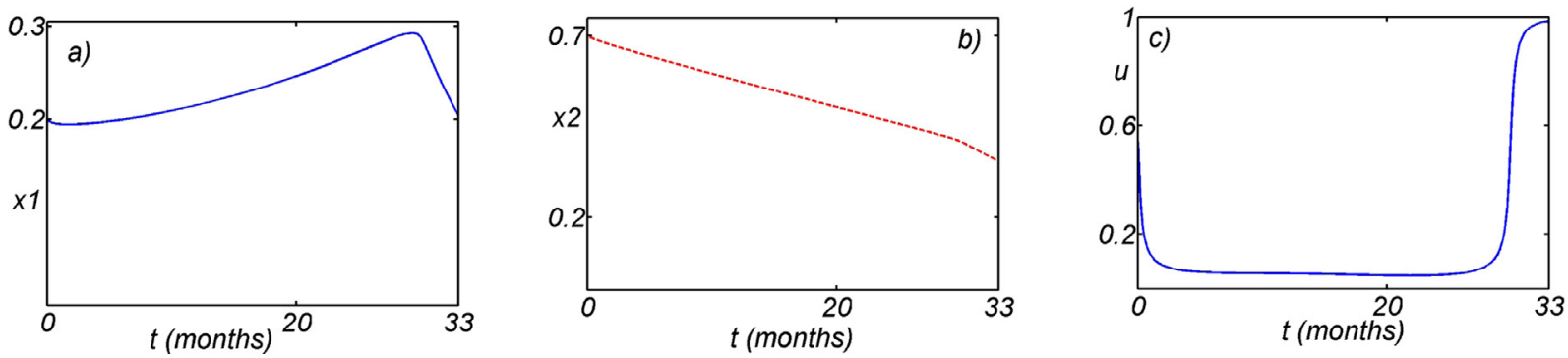

Fig. 5. Optimal solution for functional $J_{w}(u)$ in Eq. (59) with $w=w_{s}=0.0309$, and total dose $M=5$; the time horizon is $T=33$ months. Left: Tumor cells $x_{1}$. Middle: Normal cells $x_{2}$. Right: Optimal control $u^{*}(t)$ which is totally singular on $[0,1]$.

We obtain a bang-bang optimal control given by:

$$
u^{*}(t)= \begin{cases}1, & \text { for } 0 \leq t<t_{1}, \\ 0, & \text { for } t_{1} \leq t \leq T,\end{cases}
$$

and terminal states

$$
J_{1}\left(u^{*}\right)=5.74873, \quad x_{1}(T)=0.285677, \quad x_{2}(T)=0.403899 .
$$

We find $t_{1}=5$ months. Fig. 4c) shows that the strict bang-bang property holds.

$$
\phi(t)<0, \quad \forall t \in\left[0, t_{1}\right], \quad \dot{\phi}\left(t_{1}\right)>0, \quad \phi(t)>0, \quad \forall t \in\left(t_{1}, T\right] .
$$

Case $w=w_{s}=0.0309, T=33$ months.

Our computations reveal that, for the weight $w_{s}=0.0309$, the optimal control is totally singular. Thus, we have

$$
\begin{aligned}
& 0 \leq w<w_{s}: \quad u^{*}(t)=\left\{\begin{array}{lll}
0 & \text { for } & 0 \leq t \leq 28 \\
1 & \text { for } & 28 \leq t \leq 33
\end{array}\right\}, \\
& w_{s}<w \leq 1: u^{*}(t)=\left\{\begin{array}{lll}
1 & \text { for } & 0 \leq t \leq 5 \\
0 & \text { for } & 5 \leq t \leq 33
\end{array}\right\} .
\end{aligned}
$$

Hence, for $w \neq w_{s}$ all controls are bang-bang with only one switch occurring at a time when the total dose $M=5$ is reached, as depicted in Figs. 3 and 4. However, for the specific weight $w_{s}=0.0309$, the control structure changes dramatically, as shown in Fig. 5.

Moreover, our computations evidence that the strict bang-bang property (62) is satisfied for all $0 \leq w<w_{s}$, while (65) holds for $w_{s}<w \leq 1$. This explains the role of the threshold weight $w_{s}=0.0309$, where the switching function must vanish identically in $[0,1]$. Hence, for $w_{s}=0.0309$ we obtain a totally singular control in $[0,1]$, which is displayed in Fig. $5 c$ ).

It is worth mentioning that in the three obtained scenarios, depending on the value of $w$, we observe that for the threshold weight $w_{s}=0.0309$ there is a better control of the tumor cells since they remain bounded within a smaller interval, in comparison with the cases where $w<w_{s}$ or $w>w_{s}$.

Now, we increase the time horizon up to $T=66$ months:

Case $w=0, T=66$ months.

The state variables, the optimal control and the switching are shown in Fig. 6 . Similar to the case $w=0$ and $T=33$ months, initially, the dose level is zero and then jumps to the MTD during the last 5 months starting at $t_{1}=61$ months. The tumor and normal cells display a sustained growth and decrease, respectively, until the treatment starts, during which both types of cell are depleted (notice that for the normal cells there is no significant change in the trend). 

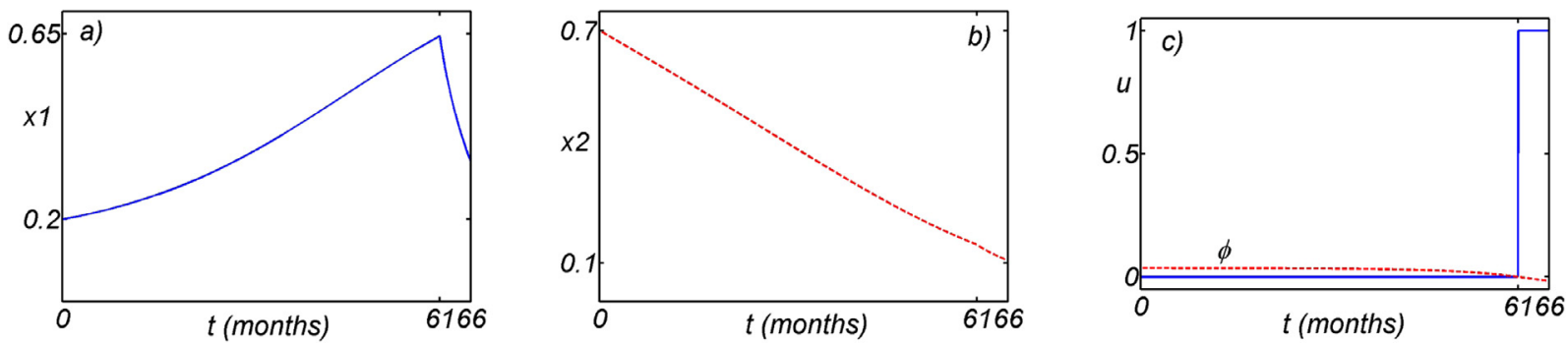

Fig. 6. Optimal solution for functional $J_{w}(u)$ in Eq. (59) with $w=0$ and total dose $M=5$; the time horizon is $T=66$ months. Left: a) Tumor cells $x_{1}$. Middle: b) Normal cells $x_{2}$. Right: c) Optimal control $u^{*}(t)$ and switching function $\phi$ satisfying the control law (43).
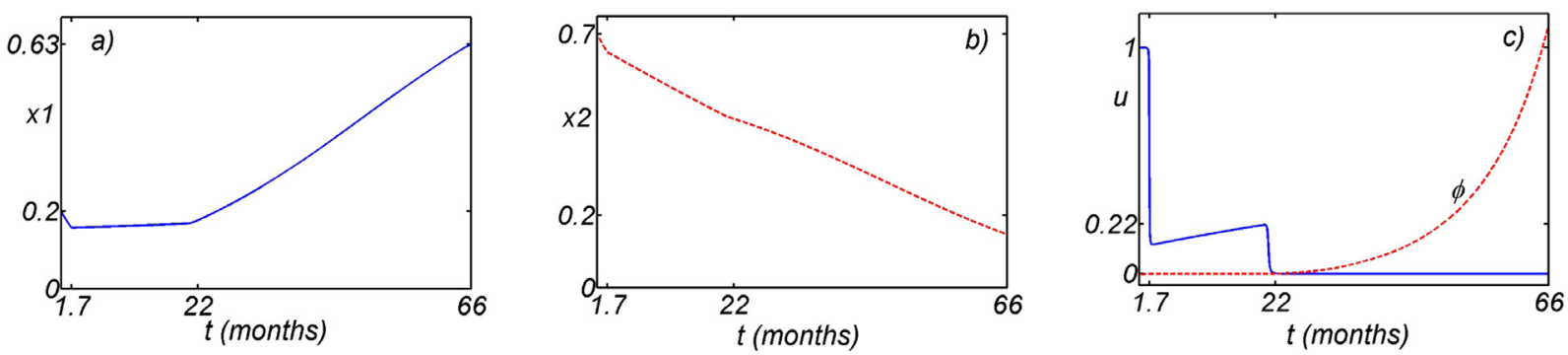

Fig. 7. Optimal solution for functional $J_{w}(u)$ in Eq. (59) with $w=1$, and total dose $M=5$; the time horizon is $T=66$ months. Left: a) Tumor cells $x_{1}$. Middle: b) Normal cells $x_{2}$. Right: c) Optimal control $u^{*}(t)$ and switching function $\phi$ satisfying the control law (43). The switching function $\phi(t)$ is zero on the singular arc $\left[t_{1}=1.716, t_{2}=22.673\right]$ months.

We obtain a bang-bang optimal control given by Eq. (60). and terminal states

$$
J_{0}\left(u^{*}\right)=0.340737, \quad x_{1}(T)=0.340737, \quad x_{2}(T)=0.106368 .
$$

In this case, we find that $t_{1}=61$ months. Fig. $6 \mathrm{c}$ ) shows that the strict bang-bang property holds.

$$
\phi(t)>0 \quad \forall t \in\left[0, t_{1}\right], \quad \dot{\phi}\left(t_{1}\right)<0, \quad \phi(t)<0 \quad \forall t \in\left(t_{1}, T\right] .
$$

Case $w=1, T=66$ months.

In this case, we obtain a control with the structure bang-singular-bang:

$$
u^{*}(t)= \begin{cases}1, & \text { for } 0 \leq t<t_{1}, \\ u_{\text {sing }}(x(t), \lambda(t)), & \text { for } t_{1} \leq t<t_{2}, \\ 0, & \text { for } t_{2} \leq t \leq T,\end{cases}
$$

The singular control $u_{\text {sing }}$ is given in Eq. (52). The found switching times are $t_{1}=1.716$ months and $t_{2}=22.6732$ months. The state and control variables are displayed in Fig. 7. The objective value and the terminal state variables are computed as:

$$
J_{1}\left(u^{*}\right)=20.903, \quad x_{1}(T)=0.630589, \quad x_{2}(T)=0.147066 .
$$

Here, the treatment starts with a full dose therapy, then it jumps to a singular dose which is followed by a terminal zero dose therapy. Thus, this type of control provides an example for a metronomic therapy, which is an alternative to MTD therapy [37,52].

We can verify numerically that the strict GLC condition (51) is satisfied not only on the singular interval [ $\left.t_{1}, t_{2}\right]$ but on the whole interval $[0, T]$. To see that the computed control $u^{*}(t)$ satisfies the necessary conditions with high accuracy, we do the following: We insert the computed values of $x(t)$ and $\lambda(t)$ into the formula (52) and check that the values of the singular control $u_{\text {sing }}(x(t), \lambda(t))$ perfectly match the computed values of $u^{*}(t)$ in the singular domain.

We have not been able to check the sufficient optimality for the solution in Fig. 7 since, to the best of our knowledge, numerically verifiable sufficient conditions are not available in the literature.

Finally, we consider the objective functional:

$$
J(u)=x_{1}(T)+\int_{0}^{T}\left(x_{1}(t)+u(t)\right) d t
$$

for the following times:

Case $T=66$ months.

Here, the treatment starts with a full dose therapy, then it jumps to a singular dose which is followed by a terminal zero dose therapy. 

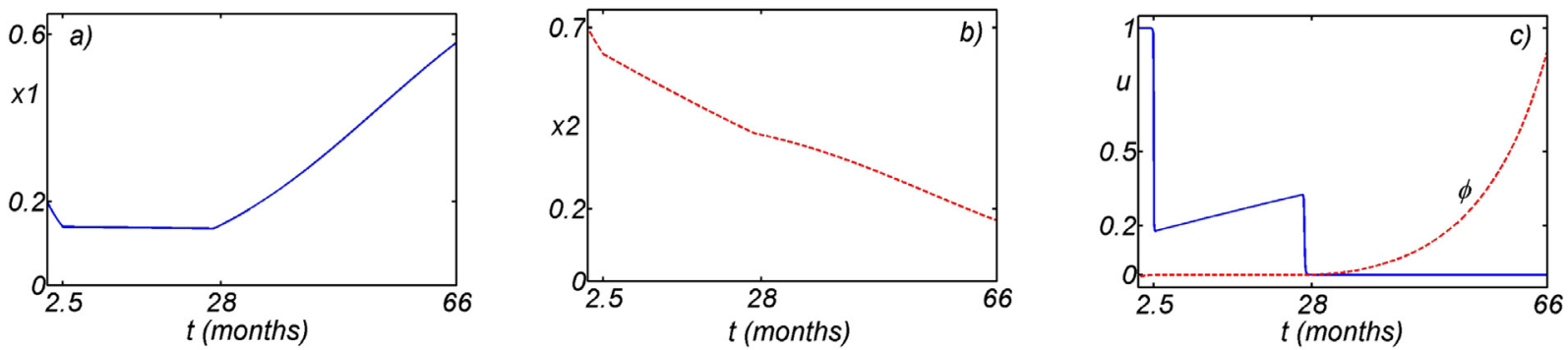

Fig. 8. Optimal solution for functional $J_{w}(u)$ in Eq. (71); the time horizon is $T=66$ months. Left: a) Tumor cells $x_{1}$. Middle: b) Normal cells $x_{2}$. Right: c) Optimal control $u^{*}(t)$ and switching function $\phi$.The switching function $\phi(t)$ is zero on the singular arc $\left[t_{1}, t_{2}\right]$
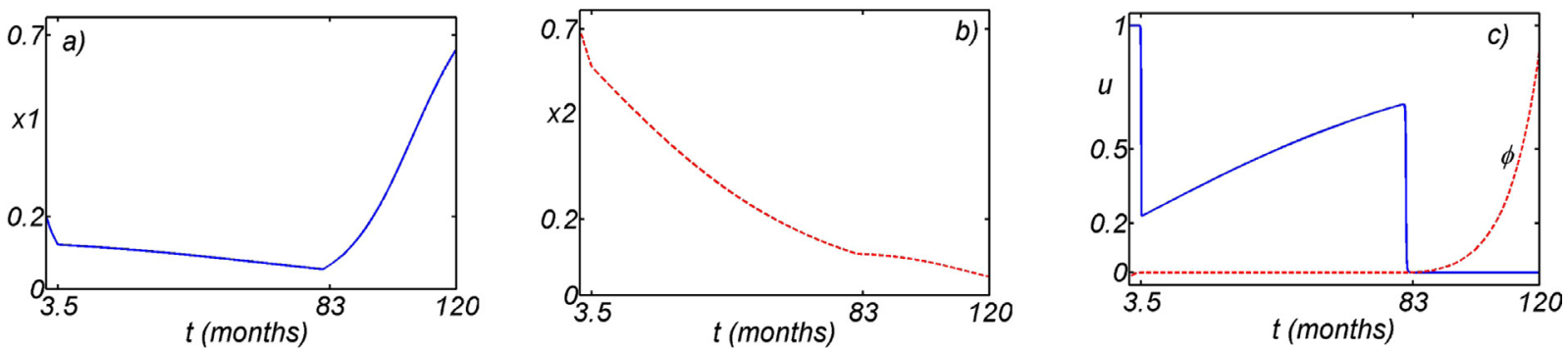

Fig. 9. Optimal solution for functional $J_{w}(u)$ in Eq. (71); the time horizon is $T=120$ months. Left: a) Tumor cells $x_{1}$, b) Middle: Normal cells $x_{2}$; Right: c) Optimal control $u^{*}(t)$ and switching function $\phi$. The switching function $\phi(t)$ is zero on the singular arc $\left[t_{1}, t_{2}\right]$.

The state variables, the optimal control and the switching are shown in Fig. 8. The switching times are computed as $t_{1}=2.54$ months and $t_{2}=28.09$ months, showing a singular arc. The objective value and the terminal state variables are computed as:

$$
J\left(u^{*}\right)=26.3415, \quad x_{1}(T)=0.579728, \quad x_{2}(T)=0.167483 .
$$

Case $T=120$ months.

Again, we obtain a control with the structure bang-singular-bang given by Eq. (69). The switching times are computed as $t_{1}=3.55$ months and $t_{2}=82.80$ months showing a long singular arc. The state and control variables are displayed in Fig. 9 . The objective value and the terminal state variables are computed as:

$$
J\left(u^{*}\right)=61.0739, \quad x_{1}(T)=0.659747, \quad x_{2}(T)=0.048251 .
$$

Here, the treatment starts with a full dose therapy, then it jumps to a singular dose with medium values which is followed by a terminal zero dose therapy. Again, we can verify numerically that the strict GLC condition (51) is satisfied on the whole interval $[0, T]$.

\section{Conclusions}

In this paper we have studied a model of slow-progressing tumor development taking into account the basic principles of tumor-normal cell interplay. The development of the equations, analysis and control problems has given rise to a theoretical framework that can be further exploited to define measurable experimental variables relevant for therapies.

Starting from a stochastic model, a deterministic approximation and a Fokker-Planck equation were derived using an expansion of the master equation or forward Kolmogorov equation. For the deterministic model, existence and uniqueness of solutions were shown and a stability analysis was carried out. Solutions of the deterministic system were calculated for values of the parameters in Table 1 , which correspond to low grade gliomas.

Using the Fokker-Planck equation, the evolution of first and second moments for the fluctuations have been derived. We have analyzed the evolution in time of the first moments (or expectations) of fluctuations near the stationary state for both populations and observed that the expectations of the fluctuations are small and tend to zero as $t \rightarrow \infty$, providing partial justification that the deterministic approximation to the stochastic model is a reasonable one.

Subsequently, we introduced an optimal control problem assuming different types of objective functions. Since the control variable appears linearly in the control system and in the objective functional, the evaluation of the necessary optimality conditions shows that optimal controls are concatenations of bang-bang and singular controls. We have obtained an explicit formula for the singular control in terms of state and adjoint variables. We have then used discretization and nonlinear programming methods to compute the control, state and adjoint variables. 
For relatively short treatment times (in terms of the natural history of low grade gliomas), we found simple bang-bang controls with only one switch which represent MTD therapies. This therapeutic modality is currently used in the clinical practice, which consists of the administration of the maximal dose of chemotherapeutical drugs that can be tolerated by the patient. We also have found an optimal control which is totally singular.

For longer treatment times we have obtained controls with a singular arc placed between two bang-bang arcs. This type of control provides an example for a metronomic therapy, which is an alternative to MTD therapy. From a practical point of view such a scheme may be more difficult to administer in the clinic, but our results provide a theoretical test bench for which realistic and easily realizable schemes can be compared and thus the efficacy of these protocols could be evaluated.

In future works, we plan to incorporate into the present model a broader hierarchy of cells (depending on their differentiation and pluripotentiality state) as well as the combination chemotherapy with neurosurgery and radiotherapy with the goal to unveil new optimal therapeutic scenarios for low grade gliomas.

\section{Acknowledgements}

This work has been supported by Ministerio de Economía y Competitividad/FEDER, Spain [grant numbers: MTM201231073 and MTM2015-71200-R], Consejería de Educación Cultura y Deporte from Junta de Comunidades de Castilla-La Mancha (Spain) [grant number PEII-2014-031-P] and James S. Mc. Donnell Foundation (USA) 21st Century Science Initiative in Mathematical and Complex Systems Approaches for Brain Cancer (Special Initiative Collaborative-Planning Grant 220020420 and Collaborative award 220020450). We thank the anonymous reviewers for helpful comments.

\section{References}

[1] Albini A, Tosetti F, Li VW, Noonan DM, Li WW. Cancer prevention by targeting angiogenesis. Nat Rev Clin Oncol 2012;9:498-509. http://dx.doi.org/10. 1038/nrclinonc.2012.120.

[2] Allen LJS. An introduction to stochastic processes with applications to biology. 2nd. Boca Raton, Florida, USA: CRC Press, Taylor \& Francis Group; 2010 978-1-4398-9468-2, http://www.taylorandfrancis.com

[3] Altizer S, Bartel R, Han BA. Animal migration and infectious disease risk. Science 2011;331:296-302. http://dx.doi.org/10.1126/science.1194694.

[4] Altrock PM, Michor F. The mathematics of cancer: integrating quantitative models. Nat Rev Cancer 2015;15:730-45. http://dx.doi.org/10.1038/nrc4029.

[5] Álvarez Arenas A, Belmonte-Beitia J, Calvo GF. Nonlinear waves in a simple model of high-grade glioma. Appl Math Nonlinear Sci 2016;1:405-22. http://dx.doi.org/10.21042/AMNS.2016.2.00035

[6] Anderson ARA. A hybrid mathematical model of solid tumour invasion: the importance of cell adhesion. Math Med Biol 2005;22(2):163-86. doi:10. 1093/imammb/dqi005.

[7] Andre N, Carre M, Pasquier E. Metronomics: towards personalized chemotherapy? Nat Rev Clin Oncol 2014;11(7):413-31. http://dx.doi.org/10.1038] nrclinonc.2014.89

[8] Andre N, Pasquier E. For cancer, seek and destroy or live and let live? Nature 2009;460(7253). 324-324 http://dx.doi.org/10.1038/460324c

[9] Badoual M, Gerin C, Deroulers C, Grammaticos B, J-F L, Oppenheim C, et al. Oedema-based model for diffuse low-grade gliomas: application to clinical cases under radiotherapy. Cell Prolif 2014;47:369-80.

[10] Bansaye V, Méléard S. Stochastic models for structured populations. Mathematical biosciences institute lecture series, 14. 1st. New York: Springer International Publishing; 2015. 987-3-319-21710-9

[11] Bell DJ, Jacobson DH. Singular optimal control problems. Academic Press London; New York; 1975. 012085060501208506050120850605

[12] Belmonte-Beitia J, Calvo GF, Pérez-García VM. Effective particle methods for Fisher-Kolmogorov equations: theory and applications to brain tumor dynamics. Commun Nonlinear Sci Numer Simulat 2014;19:3267-83. http://dx.doi.org/10.1016/j.cnsns.2014.02.004

[13] Betts JT. Practical methods for optimal control and estimation using nonlinear programming. 2nd. New York, NY, USA: Cambridge University Press; 2009. 0898716888, 9780898716887

[14] Bonnard B, Chyba M. Singular trajectories and their role in control theory. Mathematiques \& applications. Paris, New York: Springer; 2003. 3-54000838-1, http://opac.inria.fr/record=b1100529

[15] Bose T, Trimper S. Stochastic model for tumor growth with immunization. Phys Rev E 2009;79:051903. doi:10.1103/PhysRevE.79.051903.

[16] Bressloff PC. Stochastic processes in cell biology. Interdisciplinary Applied Mathematics, 41. 1st. New York: Springer International Publishing; 2014. 987-3-319-08487-9

[17] Browder T, Butterfield CE, Kräling BM, Shi B, Marshall B, O'Reilly MS, et al. Antiangiogenic scheduling of chemotherapy improves efficacy against experimental drug-resistant cancer. Cancer Res 2000;60(7):1878-86. http://cancerres.aacrjournals.org/content/60/7/1878

[18] Caiazzo A, Ramis-Conde I. Multiscale modelling of palisade formation in glioblastoma multiforme. J Theor Biol 2015;383:145-56. http://dx.doi.org/10. 1016/j.jtbi.2015.07.021.

[19] Chis OOD, Concu R. Review of stochastic stability and analysis tumor-immune systems. Curr Bioinform 2013;8(4):390-400. doi:10.2174/ 1574893611308040002 .

[20] de Pillis L, Gu W, Fister K, Head T, Maples K, Murugan A, et al. Chemotherapy for tumors: an analysis of the dynamics and a study of quadratic and linear optimal controls. Math Biosci 2007;209(1):292-315. http://dx.doi.org/10.1016/j.mbs.2006.05.003.

[21] d'Onofrio A, Ledzewicz U, Maurer H, Schattler H. On optimal delivery of combination therapy for tumors. Math Biosci 2009;222(1):13-26. http: //dx.doi.org/10.1016/j.mbs.2009.08.004.

[22] Drasdo D, Hoehme S, Block M. On the role of physics in the growth and pattern formation of multi-cellular systems: what can we learn from individual-cell based models? J Stat Phys 2007;128(1):287-345. doi:10.1007/s10955-007-9289-x.

[23] Dubin N. A stochastic model for immunological feedback in carcinogenesis: analysis and approximations. Springer-Verlag Berlin; 1976. 3540077863

[24] Durret R. Branching process models of cancer. Springer; 2015. 978-3-319-16064-1

[25] Fernández LA, Pola C. Catalog of the optimal controls in cancer chemotherapy for the gompertz model depending on pk/pd and the integral constraint. Discrete Cont Dynam Syst - Ser B 2014;19(6):1563-88. doi:10.3934/dcdsb.2014.19.1563.

[26] Fleming W, Rishel RW. Deterministic and stochastic optimal control. Applications of mathematics. New York, Berlin, Heidelberg: Springer; 1975. 3540-90155-8, http://opac.inria.fr/record=b1081427

[27] Forst DA, Nahed BV, Loeffler JS, Batchelor TT. Low-grade gliomas. The Oncologist 2014;19:403-13. http://dx.doi.org/10.1634/theoncologist.2013-0345.

[28] Gatenby RA, Gawlinski ET. A reaction-diffusion model of cancer invasion. Cancer Res 1996;56(24):5745-53. http://cancerres.aacrjournals.org/content/ 56/24/5745.abstract

[29] Gerin C, Pallud J, Grammaticos B, Mandonnet E, Deroulers C, Varlet P, et al. Improving the time-machine: estimating date of birth of grade ii gliomas. Cell Prolif 2012;45:76-90.

[30] Greaves M, Maley CC. Clonal evolution in cancer. Nature 2012;481:306-13. http://dx.doi.org/10.1038/nature10762. 
[31] JM C, M B, H H. About a generalized model of lymphoma. J Math Anal Appl 2012;386(2):813-29. doi:10.1016/j.jmaa.2011.08.043.

[32] Kampen NV. Stochastic processes in physics and chemistry. North Holland; 2007.

[33] Kerbel RS, Kamen BA. The anti-angiogenic basis of metronomic chemotherapy. Nat Rev Cancer 2004;4(6):423-36. http://dx.doi.org/10.1038/nrc1369

[34] Krener AJ. The high order maximal principle and its application to singular extremals. SIAM J Control Optimization 1977;15(2):256-93.

[35] Kuczynski EA, Sargent DJ, Grothey A, Kerbel RS. Drug rechallenge and treatment beyond progression-implications for drug resistance. Nat Rev Clin Oncol 2013;10:571-87. http://dx.doi.org/10.1038/nrclinonc.2013.158.

[36] Le Rhun E, Taillibert S, Chamberlain MC. Current management of adult diffuse infiltrative low grade gliomas. Curr Neurol Neurosci Rep 2016;16(2):110. doi:10.1007/s11910-015-0615-4.

[37] Ledzewicz U, Schättler H. A review of optimal chemotherapy protocols: from mtd towards metronomic therapy. Math Model Nat Phenom 2014;9:13152. doi: $10.1051 / \mathrm{mmnp} / 20149409$.

[38] Ledzewicz U. Drug resistance in cancer chemotherapy as an optimal control problem. Discrete Cont Dyn Syst Ser-B 2006;6.

[39] Ledzewicz U, Maurer H, Schättler H. Optimal and suboptimal protocols for a mathematical model for tumor anti-angiogenesis in combination with chemotherapy. Mathematical Biosciences and Engineering 2011;8(2):307-23.

[40] Lenhart S, Workman JT. Optimal control applied to biological models. Mathematical and computational biology. Boca Raton (Fla.), London: Chapman \& Hall/CRC; 2007. 978-1-584-88640-2, http://opac.inria.fr/record=b1123326

[41] Losick R, Desplan C. Stochasticity and cell fate. Science 2008;320:65-8. http://dx.doi.org/10.1126/science.1147888.

[42] Louis DN, Ohgaki H, Wiestler OD, Cavenee WK. WHO classification of tumours of the central nervous system. 69008 Lyon, France: International Agency for Research on Cancer; 2007. 978-92-832-2430-2

[43] Louis DN, Perry A, Reifenberger G, von Deimling A, Figarella-Branger D, Cavenee WK, et al. The 2016 world health organization classification of tumors of the central nervous system: a summary. Acta Neuropathol 2016;131(6):803-20. doi:10.1007/s00401-016-1545-1.

[44] Lukes DL. Differential equations : classical to controlled. Academic Press New York; 1982. 012459980

[45] Martínez-González A, Calvo G, Pérez-Romasanta LA, Pérez-García VM. Hypoxic cell waves around necrotic cores in glioblastoma: a biomathematical model and its therapeutic implications. Bull Math Biol 2012;74:2875-96. doi:10.1007/s11538-012-9786-1.

[46] Maurer H, Büskens C, Kim J-HR, Kaya CY. Optimization methods for the verification of second order sufficient conditions for bang-bang controls. Optimal Control Appl Methods 2005;26(3):129-56. doi:10.1002/oca.756.

[47] Merrill SJ. Stochastic models of tumor growth and the probability of elimination by cytotoxic cells. J Math Biol 1984;20(3):305-20. doi:10.1007/ BF00275990.

[48] Munsky B, Neuert G, van Oudenaarden A. Using gene expression noise to understand gene regulation. Science 2012;336:183-7. http://dx.doi.org/10. 1126/science.1216379.

[49] Nakamura T, Masuda K, Harada S, Akioka K, Sako H. Pancreatic cancer: slow progression in the early stages. Int J Surg Case Rep 2013;4:693-6. http://dx.doi.org/10.1016/j.ijscr.2013.04.040.

[50] Osmolovskii NP, Maurer H. Applications to regular and bang-bang control: second-order necessary and sufficient optimality conditions in calculus of variations and optimal control. Philadelphia, PA, USA: Society for Industrial and Applied Mathematics; 2012. 1611972353, 9781611972351

[51] Pallud J, Blonski M, Mandonne E, Audureau E, Fontaine D, Sanai N, et al. Velocity of tumor spontaneous expansion predicts long-term outcomes for diffuse low-grade gliomas. Neuro Oncol 2013;15:595-606. http://neuro-oncology.oxfordjournals.org/content/15/5/595.long

[52] Pasquier E, Kavallaris M, Andre N. Metronomic chemotherapy: new rationale for new directions. Nat Rev Clin Oncol 2010;7(8):455-65. http://dx.doi, org/10.1038/nrclinonc.2010.82

[53] Pérez-García VM, Calvo GF, Belmonte-Beitia J, Diego D, Pérez-Romasanta L. Bright solitary waves in malignant gliomas. Physical Review E 2011;84. doi:10.1103/PhysRevE.84.021921

[54] Pontryagin LS, Boltyanskii VG, Gamkrelidze RV, Mishchenko E. The mathematical theory of optimal processes (International series of monographs in pure and applied mathematics). Interscience Publishers; 1962. http://www.amazon.com/exec/obidos/redirect?tag=citeulike07-20\&path=ASIN/ B0007HRVE4

[55] Rojas C, Belmonte-Beitia J, Pérez-García VM, Maurer H. Dynamics and optimal control of chemotherapy for low grade gliomas: insights from a mathematical model. Discrete Cont Dynam Syst - Series B 2016;21(6):1895-915. doi:10.3934/dcdsb.2016028.

[56] Rong Y, Durden DL, Van Meir EG, Brat DJ. 'Pseudopalisading' necrosis in glioblastoma: a familiar morphologic feature that links vascular pathology, hypoxia, and angiogenesis. J Neuropathol \& Exp Neurol 2006;65(6):529-39. doi:10.1097/00005072-200606000-00001.

[57] Rozen WM, Joseph S, Lo PA. Spontaneous regression of low-grade gliomas in pediatric patients without neurofibromatosis. Pediatr Neurosurg 2008;44:324-8. http://www.karger.com/Article/Abstract/134925

[58] Sanai N, Chang S, Berger MS. Low-grade gliomas in adults. J Neurosurg 2011;115:948-65. http://thejns.org/doi/pdf/10.3171/2011.7.JNS10238

[59] Schättler H, Ledzewicz U. Geometric optimal control. Interdisciplinary applied mathematics, 38. Springer, New York; 2012. doi:10.1007/ 978-1-4614-3834-2. 978-1-4614-3833-5. Theory, methods and examples

[60] Swan GW. Applications of optimal control theory in biomedicine. Monographs and textbooks in pure and applied mathematics. New York: M. Dekker; 1984. 0-8247-7192-3, http://opac.inria.fr/record=b1092081

[61] Tandon A, Schiff D. Therapeutic decision making in patients with newly diagnosed low grade glioma. Curr Treat Options Oncol 2014;15:529-38. http://dx.doi.org/10.1007/s11864-014-0304-6.

[62] Wachter A, Biegler LT. On the implementation of an interior-point filter line-search algorithm for large-scale nonlinear programming. Math Program 2006;106(1):25-57. doi:10.1007/s10107-004-0559-y.

[63] Wodarz D, Komarova NL. Dynamics of cancer: mathematical foundations of oncology. 1st. River Edge, NJ, USA: World Scientific Publishing Co., Inc.; 2014. 9789814566360,9814566365 